\title{
Supporting Cell Division Is Not Required for Regeneration of Auditory Hair Cells After Ototoxic Injury In Vitro
}

\author{
Jialin Shang ${ }^{1}$, Jon Cafaro ${ }^{1}$, Rachel Nehmer ${ }^{1}$, and Jennifer Stone $^{1}$ \\ ${ }^{1}$ Department of Otolaryngology/Head and Neck Surgery, The Virginia Merrill Bloedel Hearing Research Center, University of \\ Washington School of Medicine, Seattle, WA 98195-7923, USA
}

Received: 7 August 2009; Accepted: 22 December 2009; Online publication: 18 February 2010

\begin{abstract}
In chickens, nonsensory supporting cells divide and regenerate auditory hair cells after injury. Anatomical evidence suggests that supporting cells can also transdifferentiate into hair cells without dividing. In this study, we characterized an organ culture model to study auditory hair cell regeneration, and we used these cultures to test if direct transdifferentiation alone can lead to significant hair cell regeneration. Control cultures (organs from posthatch chickens maintained without streptomycin) showed complete hair cell loss in the proximal (high-frequency) region by 5 days. In contrast, a 2-day treatment with streptomycin induced loss of hair cells from all regions by 3 days. Hair cell regeneration proceeded in culture, with the time course of supporting cell division and hair cell differentiation generally resembling in vivo patterns. The degree of supporting cell division depended upon the presence of streptomycin, the epithelial region, the type of culture media, and serum concentration. On average, $87 \%$ of the regenerated hair cells lacked the cell division marker BrdU despite its continuous presence, suggesting that most hair cells were regenerated via direct transdifferentiation. Addition of the DNA polymerase inhibitor aphidicolin to culture media prevented
\end{abstract}

Electronic supplementary material The online version of this article (doi:10.1007/s10162-009-0206-7) contains supplementary material, which is available to authorized users.

Correspondence to: Jennifer Stone · Department of Otolaryngology/ Head and Neck Surgery, The Virginia Merrill Bloedel Hearing Research Center - University of Washington School of Medicine . Seattle, WA 98195-7923, USA. Telephone: +1-206-6164108; fax: +1206-2215685; email: stoner@u.washington.edu supporting cell division, but numerous hair cells were regenerated nonetheless. These hair cells showed signs of functional maturation, including stereociliary bundles and rapid uptake of FM1-43. These observations demonstrate that direct transdifferentiation is a significant mechanism of hair cell regeneration in the chicken auditory after streptomycin damage in vitro.

Keywords: hair cell, supporting cell, regeneration, basilar papilla, BrdU, aphidicolin, direct transdifferentiation, proliferation

\section{INTRODUCTION}

Mechanosensitive hair cells (HCs) are required for hearing. HCs are injured by ototoxic drugs, intense sound stimuli, and changes associated with aging. Mature mammals cannot replace auditory HCs, but mature birds can. Avian HC regeneration occurs via division of progenitor cells within the auditory epithelium (basilar papilla, BP; Corwin and Cotanche 1988; Ryals and Rubel 1988; Raphael 1992; Hashino and Salvi 1993; Stone and Cotanche 1994). These progenitor cells, called supporting cells (SCs), are quiescent unless HCs are injured (Corwin and Cotanche 1988; Ryals and Rubel 1988; Lippe et al. 1991; Warchol and Corwin 1996). Upon HC loss, SCs divide and progeny differentiate into HCs or SCs. Normal numbers of HCs return within 2-3 weeks (reviewed in Stone and Cotanche 2007). Restoration of normal structure and function, including cellular maturation and reinnervation, takes several weeks (reviewed in Bermingham-McDonogh and Rubel 2003).

Several observations suggest that some HCs in the chicken BP may be regenerated by a process called direct transdifferentiation, which is the phenotypic con- 
version of SCs into HCs without cell division (reviewed in Morest and Cotanche 2004; Stone and Cotanche 2007). This process contributes to HC regeneration in cultured saccules of frogs and salamanders after aminoglycoside-induced injury (Baird et al. 1996, 2000; Taylor and Forge 2005). In chickens, some regenerated auditory HCs are unlabeled for a proliferation marker $\left({ }^{3} \mathrm{H}\right.$-thymidine or bromodeoxyuridine $(\mathrm{BrdU}))$ despite its continual perfusion into the inner ear after damage (Roberson et al. 1996, 2004). Cells with features of both HCs and SCs were seen in regenerating BPs, suggesting such cells were SCs converting into HCs (Adler et al. 1997; Cafaro et al. 2007). Also, attenuation of SC division did not prevent the reemergence of HCs in the BP (Adler and Raphael 1996). While this latter finding suggests HCs can be regenerated independent of SC division, inhibition of division in this study was incomplete. Therefore, despite substantial evidence for direct transdifferentiation in the chicken BP, further studies are warranted.

Here, we describe an organotypic culture system for the chicken BP that has allowed us to examine the contribution of direct transdifferentiation to auditory HC regeneration after drug damage. Three studies have previously described patterns of auditory HC loss in control and drug-treated organ cultures (Oesterle et al. 1993; Frenz et al. 1998; Cheng et al. 2003). Oesterle et al. (1993) also showed that limited SC division occurs in organ cultures maintained without ototoxic drugs for 2 days. Other studies showed that substantial SC division occurs in organ cultures after drug damage (Navaratnam et al. 1996; Daudet et al. 2009) or HC ablation (Warchol and Corwin 1996), with some mitotic events leading to production of new HCs (Navaratnam et al. 1996). In the current study, we examined the time course, levels, and spatial patterns of SC division and $\mathrm{HC}$ regeneration in cultured chicken BPs treated with streptomycin. We found that high numbers of HCs regenerated after drug damage were formed by direct transdifferentiation, even when SC division was completely blocked.

\section{METHODS}

\section{Chicken housing}

Fertile eggs of chickens (Gallus domesticus) of the White Leghorn variety were purchased from Hyline International (Graham, WA, USA) and maintained in a standard incubator until hatching. Hatchlings were housed in heated brooders with free access to water and food until the initiation of experiments, between posthatch days 5 and 10. All methods were approved by the University of Washington's IACUC and conformed to AALAC standards.

\section{Preparation of uncultured tissue}

In some studies, comparisons were made between BPs that had been cultured and uncultured BPs that had been dissected from control or gentamicin-treated chickens and immediately fixed. For these experiments, chickens received a single subcutaneous injection of gentamicin (Sigma-Aldrich, St. Louis, MI, USA) at a dose of $250 \mathrm{mg} / \mathrm{kg}$, administered on two consecutive days, and survived for various periods after gentamicin treatment. Chickens were euthanized by Nembutal overdose $(100 \mathrm{mg} / \mathrm{kg}$, intraperitoneal injection) and decapitation. The tympanic membrane and columella were dissected away, creating a hole into the inner ear. The head was then immersion-fixed in $4 \%$ paraformaldehyde for 1 to $1.5 \mathrm{~h}$. Tissue was rinsed in phosphate-buffered saline (PBS). The cochlear duct was removed from the temporal bone, and the tegmentum vasculosum and tectorial membrane were mechanically removed from the cochlear duct.

\section{Organ cultures}

Chickens were euthanized by rapid decapitation. Following brief immersion in $70 \%$ ethanol, cochlear ducts were removed via a lateral approach through the middle ear and placed in ice-cold sterile Hanks buffered saline solution (HBSS; Sigma-Aldrich). The tegmentum vasculosum was dissected off the cochlear duct, and the remaining tissue of the cochlear duct was cultured free-floating in $500 \mu \mathrm{l}$ of media per well in 48-well plates. The remaining tissues (auditory end organs) were incubated at $37^{\circ} \mathrm{C}$ in $95 \%$ air $/ 5 \% \mathrm{CO}_{2}$. For comparison, two culture media (Sigma-Aldrich) were used: Dulbecco's Modified Eagle's Medium (DMEM) or basal minimal Eagle's (BME)/Earle's buffered salt solution (EBSS; a mixture of one part BME and two parts EBSS; Oesterle et al. 1993). No agitation of tissue was performed during the culture period.

Cultures were maintained for periods ranging from 1 to 12 days. For each experiment, at least three culture runs were performed, with five to eight organs per run. In every experiment, culture media were supplemented with glucose $(4.5 \mathrm{~g} / 1$ in DMEM and $3.9 \mathrm{~g} / 1 \mathrm{in}$ BME/EBSS) and fetal bovine serum at $0 \%$, $1 \%, 5 \%$, or $10 \%$ (Atlantic Biologicals, Atlanta, GA, USA). In some experiments, the following drugs (from Sigma-Aldrich) were also added to culture media: penicillin/streptomycin solution (working concentration of 200 penicillin units per milliliter and working concentration of $78 \mu \mathrm{M}$ streptomycin), $\operatorname{BrdU}(0.1$ or $1 \mu \mathrm{M})$, dimethyl sulfoxide (DMSO) at $0.5 \%$ or $1 \%$, and/or aphidicolin $(3 \mu \mathrm{M}, 10 \mu \mathrm{M}$, or $25 \mu \mathrm{M})$. 
At the end of the culture period, the tectorial membrane was removed from the sensory epithelium using a combination of enzymatic and mechanical methods (Stone et al. 1996). Tissue was fixed with $4 \%$ buffered paraformaldehyde for $30 \mathrm{~min}$ to $1 \mathrm{~h}$ at room temperature and then rinsed in PBS.

\section{Live labeling with FM1-43-FX}

Uptake of the fixable styryl dye FM1-43-FX (Molecular Probes, Eugene, OR, USA) into live HCs was examined in two experiments. In the first experiment, aimed to label and trace original HCs during streptomycin treatment, cochlear ducts were isolated as described above; the tegmentum vasculosum was dissected off, and the tectorial membrane was removed mechanically after treatment with thermolysin $(3 \mathrm{mg} / \mathrm{ml})$. Organs were placed in room-temperature HBSS for 3 min and were transferred to FM1-43-FX for 2 min at $37^{\circ} \mathrm{C}$. Organs were transferred to culture media, with or without streptomycin, at $37^{\circ} \mathrm{C}$ and were fixed $10 \mathrm{~min}, 2$ days, or 5 days later. In the second experiment, aimed to determine if regenerated HCs rapidly incorporate FM1-43, organs that had been cultured for 10 or 12 days were rinsed in warmed HBSS and then placed in $37^{\circ} \mathrm{C}$ FM1-43-FX for $20 \mathrm{~s}$. Organs were transferred to culture media for $30 \mathrm{~min}$ and fixed. For both experiments, FM1-43-FX was dissolved in DMSO to make a 1-mM stock solution. The dye was then diluted to $5 \mu \mathrm{M}$ in HBSS, with the final concentration of DMSO at $0.5 \%$.

\section{Labeling of fixed tissues}

To assess stereociliary bundle morphology, auditory end organs were labeled for $1.5 \mathrm{~h}$ with rhodamine phalloidin (Molecular Probes) diluted to 200 units per milliliter in PBS plus $0.05 \%$ triton X100 detergent (PBT). To label cell nuclei, organs were immersed in $10 \mu \mathrm{g} / \mathrm{ml}$ propidium iodide (from Sigma-Aldrich and dissolved in PBT) for $1 \mathrm{~min}$.

Standard indirect immunofluorescence or immunohistochemistry was used to detect proteins in wholemount preparations (see Stone et al. 1996). The following primary antibodies were used: mouse antiBrdU (Becton Dickinson, Franklin Lakes, NJ, USA), rat anti-BrdU (clone BU1/75; Sera Laboratories, West Sussex, UK), rabbit antiphosphohistone H3 (antipH3; Upstate Biotechnology, Lake Placid, NY, USA), rabbit anti-myosinVI (purchased from University California San Diego, San Diego, CA, USA or from Proteus Biosciences, Ramona, CA, USA), mouse antihair-cell antigen (gift from Dr. Guy Richardson, University of Sussex, UK), mouse anti-class III Btubulin (gift from Dr. Anthony Frankfurter, University of Virginia), and mouse anticalmodulin (Sigma-
Aldrich). Secondary antibodies conjugated to fluorophores (Cy3, Alexa 488, Alexa 594, Cy5) were purchased from Molecular Probes or Jackson Laboratories (West Grove, PA, USA). Secondary antibodies conjugated to biotin were purchased from Vector Laboratories (Burlingame, CA, USA). Diaminobenzidine (DAB) labeling was performed using the horseradish-peroxidase-conjugated $\mathrm{ABC}$ reagent from Vector Laboratories (Stone et al. 1996).

The terminal deoxynucleotidyl transferase dUTP nick end labeling (TUNEL) reaction with fluorescent detection (Chemicon, Temecula, CA, USA) was used according to manufacturer's instructions to detect dying cells in whole mounts.

For all fluorescent labeling, auditory end organs were whole-mounted onto microscope slides in Vectashield (Vector Laboratories) and coverslipped. DABlabeled organs were whole-mounted onto microscope slides in 90\% glycerol in PBS and coverslipped. Others were processed for plastic sectioning, as described in Stone et al. (1996).

\section{Analysis}

Qualitative analyses. Qualitative analyses were performed with a Zeiss Axioplan microscope or with a confocal laser scanning microscope (Bio-Rad MRC 1024 or Olympus Fluoview 1000). For qualitative analysis of labeling for FM1-43-FX, various antibodies, or TUNEL, 12-16 BPs from at least two different culture runs were examined per group.

Quantitative analyses. Although each experiment was repeated at least two times, with six to eight BPs per run, we performed quantitative analyses only on BPs that were fully intact. Therefore, Ns varied across experiments, as described below.

Nonfluorescent labels. To quantify cumulative BrdU/DAB labeling in the BP, organs were cultured for 3 days in either DMEM or BME/EMSS, with or without streptomycin, with $1 \mu \mathrm{M}$ BrdU present the entire period. Images of the BP were captured on a Leica Aristoplan microscope at $\times 10$ using a SPOT Digital Camera and digital software (Diagnostic Instruments Inc., Sterling Heights, MI, USA). A montage of the entire $\mathrm{BP}$ was generated for each sample using National Institutes of Health (NIH) Object Image. The BP was subdivided into tenths, lengthwise (proximal/high frequency to distal/low frequency), and four sites were chosen in which to count BrdU-labeled nuclei: 10\% from the distal tip (called distal), 30\% from the distal tip (called middistal), 60\% from the distal tip (called midproximal), and $90 \%$ from the distal tip (called proximal; Fig. 1A). At each site, two $9,359-\mu \mathrm{m}^{2}$ regions within the BP were analyzed with a $\times 100$ oil objective 

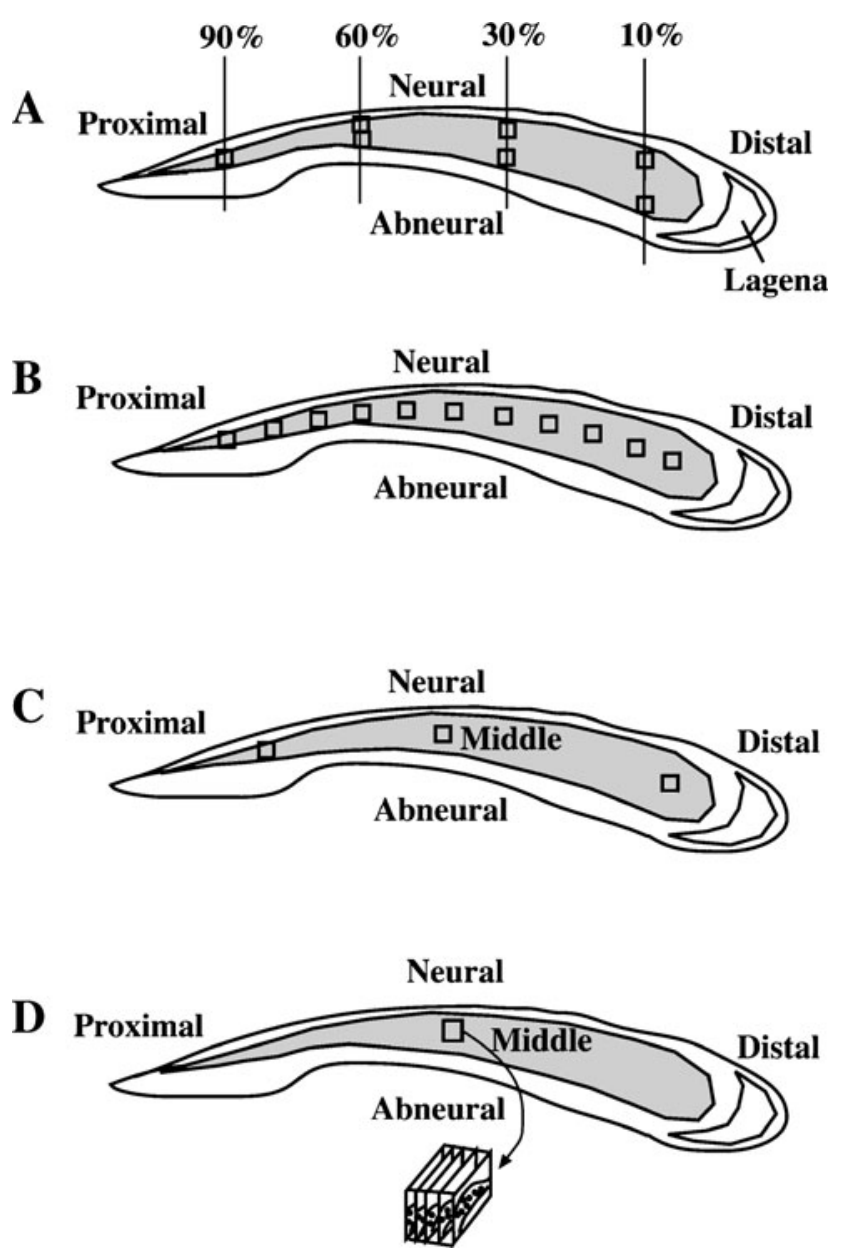

FIG. 1. Sampling methods used for quantitative analyses. These drawings depict the regions analyzed for quantitative examination of BrdU and myosinVI labeling densities. A Sampling method used for counts of BrdU labeling graphed in Figures 5 and 6 and presented in Tables 2 and 3. B Sampling method used for counts of myosinVI and BrdU labeling (discussed in text with Fig. 7 and graphed in Fig. 11). C Sampling method used for counts of myosinVI and BrdU labeling graphed in Figure 9. D Sampling method for counts of propidiumiodide-positive nuclei, discussed in the text after Figure 11.

with $\times 1.25$ zoom, one along the neural edge of the BP (called neural) and one along the abneural edge (called abneural). BrdU labeling densities (numbers of BrdUlabeled nuclei per $10,000 \mu \mathrm{m}^{2}$ ) were determined in both regions in a given site (except for the proximal site, where only the neural region was analyzed because of the narrowness of the epithelium there). A total of seven fields were sampled per BP, constituting approximately $10 \%$ of each BP's area. Only strongly labeled cells located within the BP (sensory epithelium) were counted. Three analyses were performed to obtain group data. First, we computed an average overall BrdU labeling density by averaging densities across all seven sites for all BPs in a group. These data are presented in Figure 5 and Tables 2 and 3. Second, we performed an analysis of widthwise gradients in BrdU labeling density in which data for all lengthwise sites (distal, middistal, midproximal, and proximal) for a given widthwise region (neural or abneural) were combined and averaged. For this analysis, the proximal region was omitted, since we had designated it as having only a neural region. Third, we performed an analysis of lengthwise gradients in BrdU labeling density, in which both widthwise regions (neural and abneural) for a given lengthwise site (distal, middistal, midproximal, and proximal) were combined and averaged. Data on widthwise and lengthwise gradients are presented in Table 2. Numbers of organs used for each analysis are listed in Table 1.

To estimate the percentage of BP cells that were BrdU positive after 3 days of culture in streptomycinfree media (shown in Tables 2 and 3), we performed the following analysis. First, we estimated the average density of HCs per $10,000 \mu \mathrm{m}^{2}$ in a normal BP to be 130, based on data shown in Figure 3. Second, assuming an $\mathrm{HC} / \mathrm{SC}$ ratio of 1:2 (Goodyear and Richardson 1997), we estimated that there are normally around 260 SCs per $10,000 \mu \mathrm{m}^{2}$. By dividing the BrdU labeling density for each region by 260 , we estimated the percentage of SCs that divided during the 3-day culture period. These numbers only represent rough estimates, since they do not take into account regional variations in $\mathrm{HC}$ and $\mathrm{SC}$ densities throughout the BP.

To quantify SCs in S phase at different times after damage, streptomycin-treated cultures maintained in DMEM with $10 \%$ fetal bovine serum (FBS) were pulselabeled for $4 \mathrm{~h}$ with $1 \mu \mathrm{M}$ BrdU on days $1,2,3,5$, or 7 in vitro and fixed. BrdU labeling densities were assessed using the same methods described above for cumulative BrdU labeling. Overall BrdU labeling densities are shown in Figure 6. For each time point, we analyzed four BPs (with the exception being for 2 days in vitro, for which five BPs were analyzed).

\section{TABLE 1}

Numbers of auditory end organs analyzed for cumulative BrdU labeling densities for 3-day cultures with or without streptomycin

\begin{tabular}{lrlr}
\hline Media & [Serum] & Streptomycin? & Number \\
\hline DMEM & $10 \%$ & $\mathrm{Y}$ & 11 \\
& $5 \%$ & $\mathrm{Y}$ & 4 \\
& $1 \%$ & $\mathrm{Y}$ & 6 \\
& $10 \%$ & $\mathrm{~N}$ & 12 \\
& $5 \%$ & $\mathrm{~N}$ & 3 \\
BME/EBSS & $1 \%$ & $\mathrm{~N}$ & 3 \\
& $10 \%$ & $\mathrm{Y}$ & 10 \\
& $5 \%$ & $\mathrm{Y}$ & 3 \\
& $1 \%$ & $\mathrm{Y}$ & 4 \\
& $10 \%$ & $\mathrm{~N}$ & 16 \\
& $5 \%$ & $\mathrm{~N}$ & 4 \\
& $1 \%$ & $\mathrm{~N}$ & 4 \\
\hline
\end{tabular}

$Y$ yes, $N$ no 
TABLE 2

BrdU labeling densities at 3 days in vitro

\begin{tabular}{|c|c|c|c|c|c|c|c|}
\hline \multicolumn{8}{|c|}{ No Streptomycin } \\
\hline \multicolumn{3}{|c|}{ Overall Analysis } & \multicolumn{5}{|c|}{ Width-wise Gradient Analysis } \\
\hline \multirow[t]{2}{*}{ DMEM } & \multirow{2}{*}{\multicolumn{2}{|c|}{ BME/EBSS }} & \multicolumn{2}{|l|}{ DMEM } & \multicolumn{3}{|c|}{ BME/EBSS } \\
\hline & & & $\mathbf{N}$ & $\mathbf{A N}$ & \multicolumn{2}{|c|}{$\mathbf{N}$} & $\mathbf{A N}$ \\
\hline $\begin{array}{c}5.55 \pm 8.87 \\
2.1 \%\end{array}$ & \multicolumn{2}{|c|}{$\begin{array}{c}6.16 \pm 12.8 \\
2.4 \%\end{array}$} & $\begin{array}{c}3.78 \pm 6.26 \\
1.45 \%\end{array}$ & $\begin{array}{c}3.92 \pm 7.75 \\
1.5 \%\end{array}$ & $\begin{array}{r}0.51 \\
0 .\end{array}$ & $\begin{array}{l}1.43 \\
\%\end{array}$ & $\begin{array}{c}4.30 \pm 8.52 \\
1.7 \%\end{array}$ \\
\hline \multicolumn{3}{|c|}{$\mathrm{p}=0.711$} & \multicolumn{2}{|c|}{$\mathrm{p}=0.934$} & \multicolumn{3}{|c|}{$\mathrm{p}=0.003$} \\
\hline \multicolumn{8}{|c|}{ Length-wise Gradient Analysis } \\
\hline \multicolumn{4}{|c|}{ DMEM } & \multicolumn{4}{|c|}{ BME/EBSS } \\
\hline $90 \%(\mathbf{P})$ & $60 \%$ & $30 \%$ & $10 \%(\mathrm{D})$ & $90 \%(\mathbf{P})$ & $60 \%$ & $30 \%$ & $10 \%(D)$ \\
\hline $\begin{array}{c}15.5 \pm 12.05 \\
6.0 \%\end{array}$ & $\begin{array}{c}10.1 \pm 8.85 \\
3.9 \%\end{array}$ & $\begin{array}{c}1.21 \pm 2.85 \\
0.5 \%\end{array}$ & $\begin{array}{c}0.10 \pm 0.45 \\
0 \%\end{array}$ & $\begin{array}{c}28.7 \pm 18.0 \\
11 \%\end{array}$ & $\begin{array}{c}6.42 \pm 9.35 \\
2.5 \%\end{array}$ & $\begin{array}{c}0.74 \pm 3.42 \\
0.3 \%\end{array}$ & $\begin{array}{c}0.06 \pm 0.27 \\
0 \%\end{array}$ \\
\hline
\end{tabular}

\begin{tabular}{|c|c|c|c|c|c|c|c|}
\hline \multicolumn{8}{|c|}{ Plus Streptomycin } \\
\hline \multicolumn{3}{|c|}{ Overall Analysis } & \multicolumn{5}{|c|}{ Width-wise Gradient Analysis } \\
\hline \multirow[t]{2}{*}{ DMEM } & \multirow{2}{*}{\multicolumn{2}{|c|}{ BME/EBSS }} & \multicolumn{2}{|l|}{ DMEM } & \multicolumn{3}{|c|}{ BME/EBSS } \\
\hline & & & $\mathbf{N}$ & $\mathbf{A N}$ & \multicolumn{2}{|c|}{$\mathbf{N}$} & $\mathbf{A N}$ \\
\hline $\begin{array}{c}11.6 \pm 10.3 \\
4.4 \%\end{array}$ & \multicolumn{2}{|c|}{$\begin{array}{c}6.11 \pm 9.06 \\
2.4 \%\end{array}$} & $\begin{array}{c}9.23 \pm 7.83 \\
3.6 \%\end{array}$ & $\begin{array}{c}12.3 \pm 10.8 \\
4.7 \%\end{array}$ & $\begin{array}{r}0.67 \\
0 \\
\end{array}$ & $=1.36$ & $\begin{array}{c}7.09 \pm 8.61 \\
2.7 \%\end{array}$ \\
\hline \multicolumn{3}{|c|}{$\mathrm{p}=0.000$} & \multicolumn{2}{|c|}{$\mathrm{p}=0.213$} & \multicolumn{3}{|c|}{$\mathrm{p}=0.000$} \\
\hline \multicolumn{8}{|c|}{ Length-wise Gradient Analysis } \\
\hline \multicolumn{4}{|c|}{ DMEM } & \multicolumn{4}{|c|}{ BME/EBSS } \\
\hline $90 \%(\mathbf{P})$ & $60 \%$ & $30 \%$ & $10 \%(\mathrm{D})$ & $90 \%(\mathbf{P})$ & $60 \%$ & $30 \%$ & $10 \%(\mathrm{D})$ \\
\hline $\begin{array}{c}21.7 \pm 11.6 \\
8.4 \%\end{array}$ & $\begin{array}{c}13.3 \pm 8.39 \\
5.1 \%\end{array}$ & $\begin{array}{c}10.3 \pm 6.87 \\
4.0 \%\end{array}$ & $\begin{array}{c}8.72 \pm 12.3 \\
3.35 \%\end{array}$ & $\begin{array}{c}19.5 \pm 9.20 \\
7.5 \%\end{array}$ & $\begin{array}{c}7.65 \pm 9.52 \\
2.9 \%\end{array}$ & $\begin{array}{c}2.94 \pm 5.63 \\
1.1 \%\end{array}$ & $\begin{array}{c}1.07 \pm 1.39 \\
0.4 \%\end{array}$ \\
\hline
\end{tabular}

The average number of BrdU-labeled nuclei per 10,000 $\mu \mathrm{m} 2 \pm$ S.D. is shown for BPs cultured with either no streptomycin (top) or plus streptomycin (bottom) and in either DMEM or BME/EBSS containing 10\% FBS. Data are also expressed as a percentage of total SCs (assumed to be 260 SCs per $10,000 \mu \mathrm{m} 2$ ). For the Overall Analysis, all regions were combined to obtain an average. For the Width-wise Gradient Analysis, data from the proximal (90\%) region were omitted, and data from the remaining three length-wise regions were combined to obtain an average for each width-wise region. For the Length-wise Gradient Analyses, data from the two width-wise sites were combined to obtain an average for each length-wise site. $\mathrm{N}=$ neural, $\mathrm{AN}=$ abneural. $\mathrm{P}=$ proximal, $\mathrm{D}=$ distal. $\mathrm{p}$ values apply to ANOVA

Fluorescent labels. Images for quantification of fluorescent labels were generated using confocal laser scanning microscopy (Bio-Rad MRC 1024 or Olympus Fluoview 1000).

To measure rhodamine phalloidin labeling densities, we took images from similar regions as described above for cumulative BrdU labeling (90\%, 60\%, 30\%, and
$10 \%$ lengthwise; neural and abneural widthwise) on a Zeiss Axioplan epifluorescence microscope. The area of each region was $10,000 \mu \mathrm{m}^{2}$. Phalloidin-positive bundles were counted using Image Cell Counter in three BPs for each group. Data were converted to bundles per $10,000 \mu^{2}$. Overall averages for each treatment group are shown in Figure 3.

TABLE 3

\begin{tabular}{|c|c|c|c|c|c|}
\hline \multicolumn{6}{|c|}{ Effects of serum concentration on BrdU labeling density } \\
\hline Drug condition & Media & No FBS (serum-free) & $1 \%$ FBS & $5 \%$ FBS & $10 \%$ FBS \\
\hline No Strept (control) & DMEM & - & $\begin{array}{l}8.21 \pm 11.5 \\
3.2 \%\end{array}$ & $\begin{array}{l}6.73 \pm 12.0 \\
2.6 \%\end{array}$ & $\begin{array}{l}5.55 \pm 8.87 \\
2.1 \%\end{array}$ \\
\hline & BME/EBSS & - & $\begin{array}{l}5.13 \pm 9.02 \\
2.0 \%\end{array}$ & $\begin{array}{l}4.62 \pm 9.31 \\
1.8 \%\end{array}$ & $\begin{array}{l}6.16 \pm 12.8 \\
2.4 \%\end{array}$ \\
\hline Plus Strept & DMEM $^{\mathrm{a}}$ & $20.2 \pm 11.07 .8 \%$ & $\begin{array}{l}17.6 \pm 16.2 \\
6.8 \%\end{array}$ & $\begin{array}{l}19.1 \pm 16.5 \\
7.4 \%\end{array}$ & $\begin{array}{l}11.6 \pm 10.3 \\
4.4 \%\end{array}$ \\
\hline & BME/EBSS & - & $\begin{array}{l}6.33 \pm 8.35 \\
2.4 \%\end{array}$ & $\begin{array}{l}8.30 \pm 14.2 \\
3.2 \%\end{array}$ & $\begin{array}{l}6.11 \pm 9.06 \\
2.4 \%\end{array}$ \\
\hline
\end{tabular}

The average number of BrdU-labeled nuclei per $10,000 \mu \mathrm{m}^{2} \pm \mathrm{SD}$ is shown for each type of culture media and each level of serum concentration. In addition, data are also expressed as a percentage of total SCs (assumed to be 260 SCs per 10,000 $\mu \mathrm{m}^{2}$ )

- not analyzed

${ }^{a}$ This media type alone showed statistically significant differences across serum concentration by ANOVA ( $\left.p \leq 0.05\right) ; 0 \%$ FBS (no serum, N=7 BPs), $1 \%$ FBS, and $5 \%$ FBS were significantly different from $10 \%$, but they were not different from one another 
For studies in which SC division was inhibited long term with aphidicolin (Fig. 11), we quantified myosinVI-positive and/or BrdU-positive cells in BPs cultured for 8 days (2 days plus streptomycin/ 6 days without streptomycin) with $1 \mu \mathrm{M}$ BrdU in either DMEM alone ( $n=4 \mathrm{BPs}$ ), DMEM plus DMSO ( $n=3 \mathrm{BPs}$ ), or DMEM plus aphidicolin ( $n=4$ BPs), with $1 \%$ FBS present in all cases. For each organ, we captured a Zseries stack image $\left(\times 60\right.$ oil objective, $41,209 \mu \mathrm{m}^{2}$ area per field, 1- $\mu \mathrm{m}$ steps) in 10-12 sites along the length of the BP (Fig. 1B), sampling $65-78 \%$ of the BP's area. Each stack extended from the lumenal surface to the basilar membrane. The first stack was collected at the proximal tip, and subsequent stacks were collected every other field, until the distal end was reached. Stacks were collected only in the widthwise middle region (halfway between the neural and abneural edges of the BP). Using NIH ImageJ Cell Counter, we counted cells that were myosinVI positive, BrdU positive, or doublelabeled within the stack. Data from all sites were pooled to obtain average densities (number of cells per $10,000 \mu^{2}$ ) for each label in each BP. Data were then averaged across BPs. To be considered BrdU positive, strong discrete nuclear labeling had to be present; to be considered myosinVI positive, cells had to have strong labeling throughout the cytoplasm.

To quantify myosinVI-positive or BrdU-positive cells in 8-day cultures (2 days plus streptomycin/6 days without streptomycin) grown in DMEM supplemented with $1 \%$ FBS and BrdU at different concentrations (Fig. 9), we analyzed six BPs at $0 \mu \mathrm{M}$ BrdU, five BPs at $0.10 \mu \mathrm{M} \mathrm{BrdU}$, and four BPs at $1 \mu \mathrm{M}$ BrdU, each of which was double-labeled for myosinVI and BrdU. For each BP, we generated a Z-series stack $(\times 60$ oil objective, 41,209 $\mu \mathrm{m}^{2}$ area per field, 1- $\mu \mathrm{m}$ steps) in three lengthwise sites (proximal tip, middle, distal tip), with each site located about midway between the neural and abneural edges of the BP (Fig. 1C). Each stack extended from the lumenal space through the basilar membrane. Using NIH ImageJ Cell Counter, we counted cells that were myosinVI positive, BrdU positive, or double-labeled in each stack. Data from the three sites were pooled to obtain average densities for each label for each BP (numbers of labeled cells per $10,000 \mu \mathrm{m}^{2}$ ).

All phosphohistone-H3-positive cells per BP were counted in 8-day cultures (2 days plus streptomycin/ 6 days without streptomycin) grown in DMEM supplemented with $1 \%$ FBS and either DMSO or aphidicolin by systematically scanning the entire BP. Data from four BPs were averaged for each group.

We estimated the percentage of directly transdifferentiated HCs that incorporated FM1-43 in BPs cultured for 12 days ( 2 days plus streptomycin followed by 10 days without streptomycin) with $10 \mu \mathrm{M} \mathrm{AraC}$ present the whole time. We randomly selected two regions in each of three BPs for analysis at $\times 40$ magnification and scored myosinVI-positive cells as FM1-43 positive or negative. Strong perinuclear FM1-43 signal was required for assignment as FM1-43 positive.

To estimate the density of propidium iodide-labeled nuclei in BPs cultured for 8 days (2 days plus streptomycin/ 6 days without streptomycin) in DMEM and 1\% FBS plus either DMSO or aphidicolin, we created a Zseries stack using $0.2-\mu \mathrm{m}$ steps in the middle region of the BP using a $\times 60$ oil objective (Fig. 1D). Each stack extended from the lumenal space through the basilar membrane. Each field was centered on the same landmark - the abneural edge of the superior cartilaginous plate. Using NIH Image J, five different 5 - $\mu$ m-thick optical vertical sections were generated through each volume, and brightest point projections were created. Propidium-iodide-positive nuclei in the BP (HCs and SCS) were counted in each slice; each slice's volume was originally $14,210 \mu^{3}$. For each BP, data were averaged across the five slices. Data were then averaged across BPs ( $n=4$ per group) and converted to derive the number of propidium-iodide-labeled cells per $10,000 \mu \mathrm{m}^{3}$.

Statistical Analyses. All data were expressed as averages plus or minus one standard deviation (SD). For all statistical analyses, data were subjected to analyses of variance with Fishers' protected least significant difference (Statview, Abacus Concepts). Differences were considered statistically significant when $p \leq 0.05$.

\section{RESULTS}

Patterns of in vitro hair cell loss in control and streptomycin-treated organ cultures

In initial experiments, cultures were maintained in BME/EBSS or DMEM plus 10\% FBS for various periods. Stereociliary bundles were assessed using rhodamine phalloidin, a label for filamentous actin. In control BPs (not cultured), bundles appeared evenly distributed in all regions (Fig. 2A-C). Average densities of phalloidin-positive bundles varied across regions, with lowest levels proximally and highest levels distally (Fig. 3A). In BPs cultured for 1 day without streptomycin, there was significant loss of bundles from proximal and midproximal regions (Fig. 3B). After 2 days of culture without streptomycin, significant bundle loss was evident in all regions, except the distal region (Figs. 2D-F and 3D). After 5 days of culture without streptomycin, nearly all bundles had been lost from the proximal region, but many bundles were retained in middle and distal regions (Fig. 2G-I). These trends were observed in BPs cultured in either media type. However, bundle 

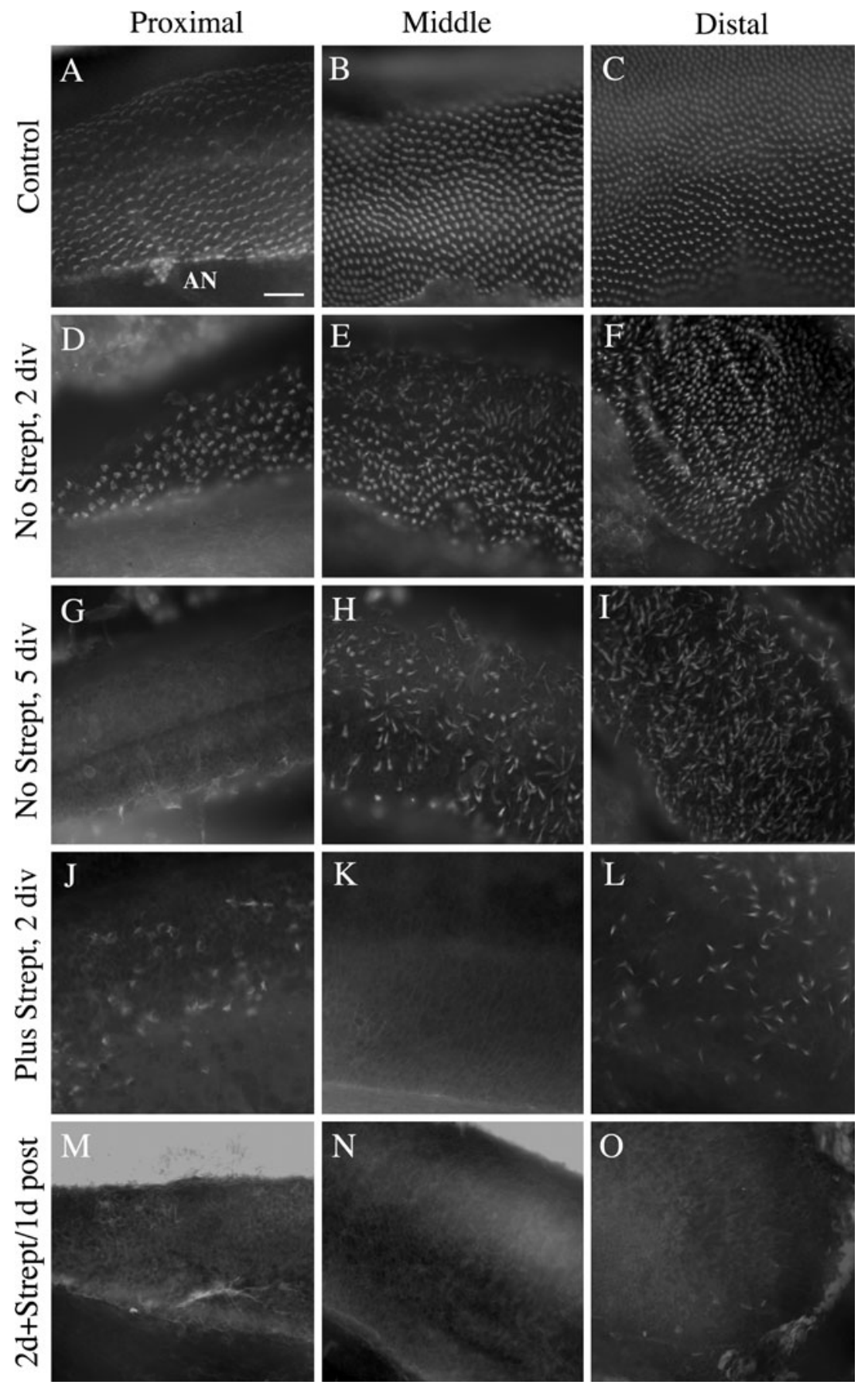

FIG. 2. Spontaneous and streptomycininduced loss of stereociliary bundles in culture. This figure shows fluorescence images of rhodamine phalloidin labeling taken from three regions of the $\mathrm{BP}$ (proximal A, D, G, J, $\mathbf{M}$; middle $\mathbf{B}, \mathbf{E}, \mathbf{H}$, $\mathbf{K}, \mathbf{N}$; and distal $\mathbf{C}, \mathbf{F}, \mathbf{I}, \mathbf{L}, \mathbf{O})$. Control BPs (not cultured) are shown in $\mathbf{A}-\mathbf{C}$. Also shown are BPs cultured in DMEM plus $10 \%$ FBS, without streptomycin (Strept) for 2 (D-F) or 5 days in vitro (div; $\mathbf{G}-\mathbf{I}$ ), and BPs cultured in DMEM plus 10\% FBS with $78 \mu \mathrm{M}$ streptomycin for $2 \operatorname{div}(\mathbf{J}-\mathbf{L})$ or with $78 \mu \mathrm{M}$ streptomycin for 2 days (d) followed by 1 day without streptomycin (M-O). Note the complete loss of rhodamine phalloidin-labeled bundles in cultures lacking streptomycin from the proximal BP by $5 \operatorname{div}(\mathbf{G})$ and the complete loss of bundles in cultures containing streptomycin from all regions of the BP by 3 div $(\mathbf{M}-\mathbf{O})$. In all images, proximal is toward the left and abneural (AN) is down. Scale bar in $\mathbf{A}=50 \mu \mathrm{m}$ for all panels. loss appeared to be slower and less extensive in BPs maintained in BME/EBSS compared to DMEM.

To assess the pattern and timing of HC bundle loss after in vitro treatment with the aminoglycoside, streptomycin, auditory end organs were cultured in BME/EBSS or DMEM plus 10\% FBS, with $78 \mu \mathrm{M}$ streptomycin (plus penicillin) for different periods. As above, phalloidin labeling was used to assess HC bundles. After 1 day of streptomycin treatment, there was bundle disruption and decreased bundle densities in all regions (Fig. 3C). After 2 days of streptomycin treatment, virtually all HC bundles were lost from the middle region, while some bundles were retained in proximal and distal regions (Figs. 2J-L and 3E). In organs cultured with streptomycin for 2 days followed by 1 day without streptomycin in either media type, no original bundles remained in the entire BP (Fig. 2M-O). We detected no clear widthwise (neural-abneural) gradients in HC loss in BPs cultured in either media type. These trends were observed in BPs cultured in either media type.

To determine if the disappearance of HC bundles reflected complete extrusion of $\mathrm{HC}$ bodies from the $\mathrm{BP}$, auditory end organs were immunolabeled to 


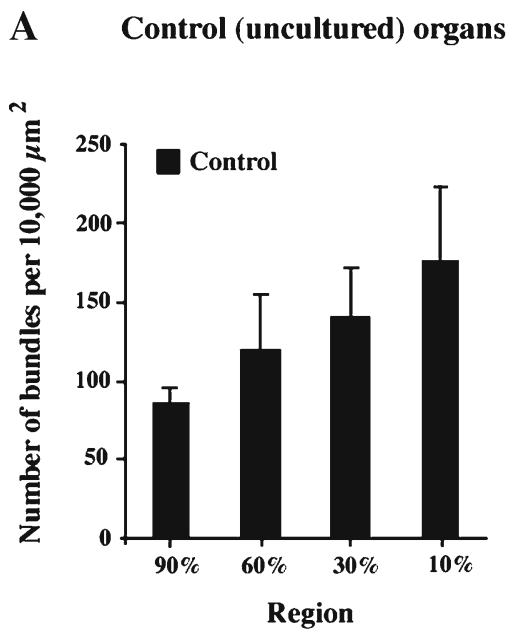

\section{B Organs cultured for 1 day No streptomycin}

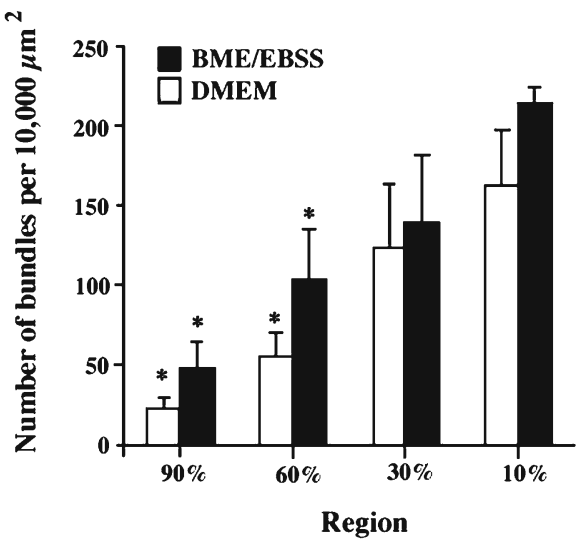

\section{Organs cultured for 2 days No streptomycin}

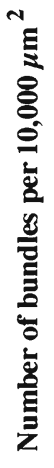

C

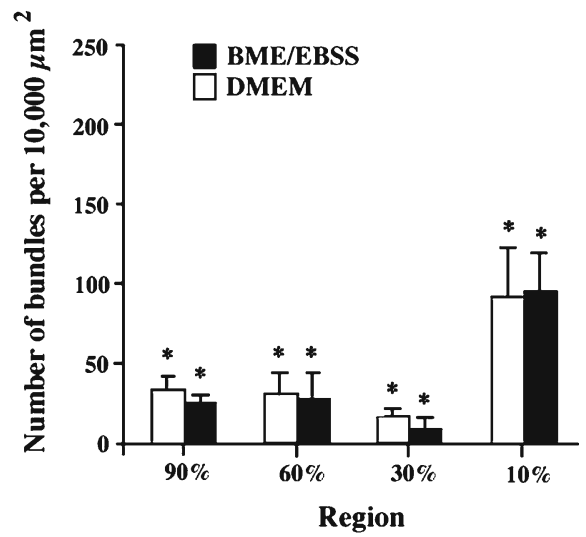

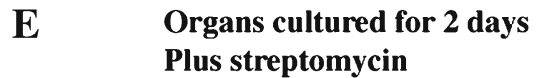

N

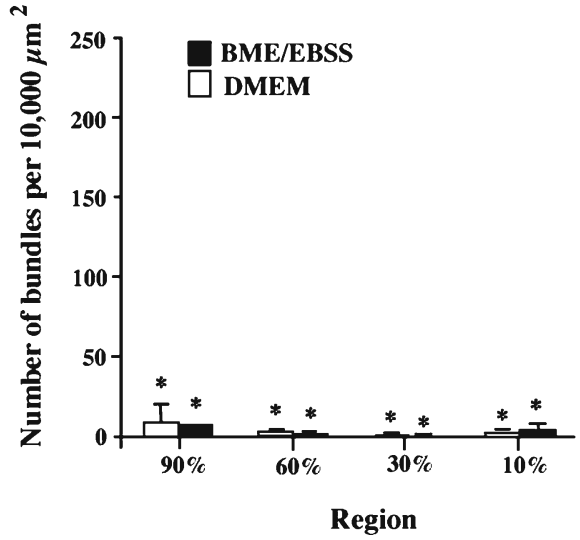

FIG. 3. Quantitative analysis of stereociliary bundle loss in culture. Average densities of $\mathrm{HC}$ bundles are graphed for control (uncultured) BPs (A), BPs cultured for 1 day with no streptomycin in DMEM or BME/ EBSS (B), BPs cultured for 1 day plus streptomycin in DMEM or BME/ EBSS (C), BPs cultured for 2 days with no streptomycin in DMEM or BME/EBSS (D), and BPs cultured for 2 days plus streptomycin in DMEM

detect the HC-specific antigen, myosinVI. In organs dissected from control chicks and fixed without culturing, strong myosinVI labeling normally occurs in $\mathrm{HC}$ cytoplasm and stereocilia but not in nonsensory SCs (Duncan et al. 2006; Fig. 4A, C). In organs cultured for 2 days with $78 \mu \mathrm{M}$ streptomycin followed by 1 day without streptomycin in DMEM plus $10 \%$ FBS, the BP was either devoid of myosinVI labeling or contained only small myosinVI-positive HC fragments (Fig. 4B, D). A similar result was obtained using antibodies to class III B-tubulin or calmodulin (data not shown), which are additional HC-selective markers (Stone et al. 1996). A similar degree of HC loss was seen in cultures grown in BME/EBSS, $10 \% \mathrm{FBS}$, and streptomycin for a similar period (data not shown).

In theory, streptomycin treatment could cause changes leading to reduced immunolabeling for some HC markers and give the false impression of HC loss.
To address this, we examined the fate of original HCs using the vital fixable dye, FM1-43-FX. Short in vitro exposures to this dye result in its selective uptake by HCs (Gale et al. 2001; Meyers et al. 2003; Si et al. 2003). BPs loaded with FM1-43-FX for $2 \mathrm{~min}$ and maintained in culture for $10 \mathrm{~min}$ in DMEM/10\% FBS without streptomycin showed strong labeling in HCs (Fig. 4E) but no significant labeling in SCs (data not shown). HC-specific FM1-43 labeling was retained when cultures were grown for an additional 2 days without streptomycin (Fig. 4F), although labeling became more condensed. Strong FM1-43-FX labeling was also retained after 5 days of growth in streptomycin-free media after loading (data not shown). These findings demonstrate that 2-min exposures to FM1-43-FX lead to selective uptake in HCs, and FM1-43-FX is retained when tissue is not exposed to ototoxic drugs. BPs were loaded with FM1-43-FX as just 

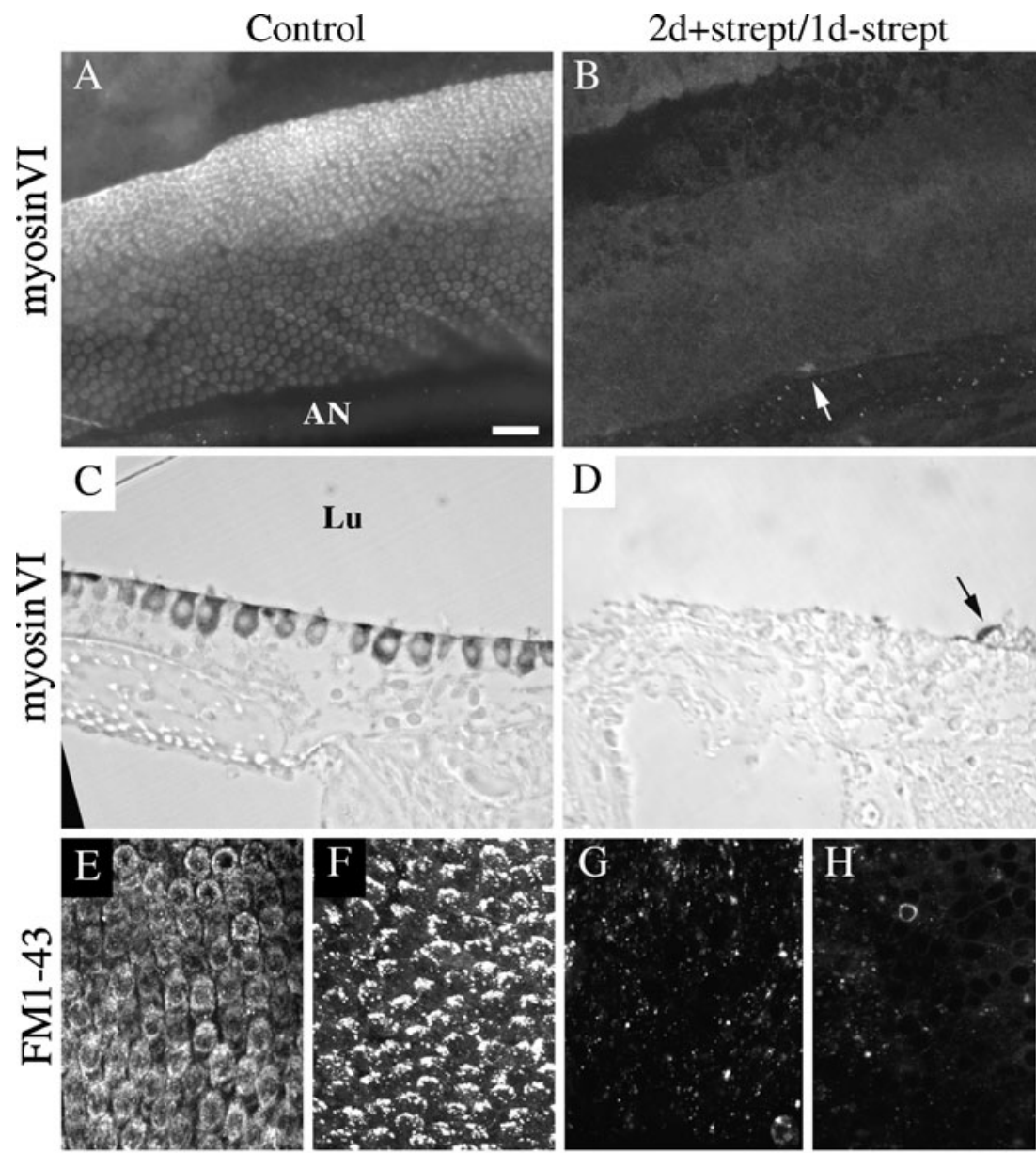

Control/HCL

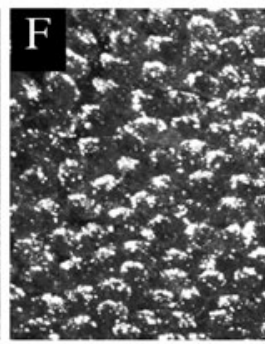

2d-strept/HCL

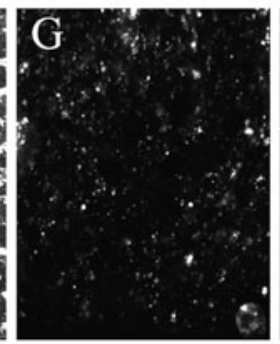

$2 \mathrm{~d}+$ strept/HCL

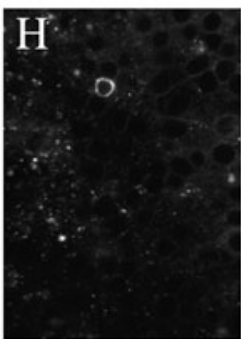

$2 \mathrm{~d}+$ strept/SCL
FIG. 4. Streptomycin treatment in culture causes loss of all hair cells from the epithelium. Panels A-D show images of myosinVI labeling in control BPs (not cultured, A, C) and in BPs cultured for 2 days in DMEM with $10 \%$ FBS with $78 \mu \mathrm{M}$ streptomycin followed by 1 day in the same media without streptomycin (B, D). Images were taken midway between the proximal and distal tips of the BP in whole-mount preparations (A, B) and plastic cross sections $(\mathbf{C}, \mathbf{D})$. Note the virtually complete loss of myosin $\mathrm{VI}$ immunoreactivity from this region in streptomycin-treated BPs (B, D). One remaining myosin $\mathrm{VI}$-positive cell is evident in B (arrow), and a single myosin VIpositive cell in the process of extrusion is shown in D (arrow). Panels $\mathbf{E}-\mathbf{H}$ show images of FM1-43-FX incorporation in the control BP (E), in a BP cultured for 2 days without streptomycin (F), and in a BP cultured for 2 days plus streptomycin (G, $\mathbf{H})$. Whole mounts from the middistal region are shown. The plane of focus is in the $\mathrm{HC}$ layer $(\mathrm{HCL})$ in $\mathbf{E}-\mathbf{G}$ and in the $\mathrm{SC}$ layer $(S C L)$ in $\mathbf{H}$. Note the retention of FM143-FX-positive HC after 2 days of culture in streptomycin-free conditions (F) compared to the complete loss of FM1-43-FX-positive HCs after 2 days of culture with streptomycin (G). FM1-43-FX-positive cells were not present in the SCL in either culture condition (H). AN abneural; Lu lumen. Scale bar in $\mathbf{A}=50 \mu \mathrm{m}$ for $\mathbf{A}$ and $\mathbf{B}$ and $20 \mu \mathrm{m}$ for C-H. described and cultured in DMEM/10\% FBS for 2 days with $78 \mu \mathrm{M}$ streptomycin. This treatment resulted in near-complete loss of FM1-43 signal from the HC layer (Fig. 4G), although some diffuse punctate signal was sometimes seen, perhaps in dying HCs or HC remnants. The SC layer continued to show negligible FM1-43 labeling (Fig. 4H).

Collectively, these experiments indicate that 2-day in vitro treatments with $78 \mu \mathrm{M}$ streptomycin cause nearcomplete loss of auditory HCs by 1 day later. Given the advantage of ridding the $\mathrm{BP}$ of all original $\mathrm{HCs}$ for regeneration studies, we used 2-3-day treatments with $78 \mu \mathrm{M}$ streptomycin to induce auditory HC loss in most experiments described below. However, we also performed some experiments with brief, 2-h treatments of $78 \mu \mathrm{M}$ streptomycin, using phalloidin to mark $\mathrm{HC}$ bundles (Supplementary Fig. 1). These shorter treatments caused a proximal-to-distal gradient of bundle loss, which resembled what has been reported after aminoglycoside administration in vivo (e.g., Janas et al. 1995) or short aminoglycoside exposures in vitro (e.g., Cheng et al. 2003). Bundles from original HCs were retained in middle and proximal regions as late as 5 days after brief streptomycin treatments.
Supporting cell proliferation occurs in basilar papillae cultured without streptomycin

To determine if spontaneous $\mathrm{HC}$ loss in cultures grown without streptomycin triggers SC division, we examined uptake of the nucleoside analog bromodeoxyuridine (BrdU), a marker of cells that enter the DNA synthesis (S) phase of the cell cycle (Gratzner $1982)$. BrdU $(1 \mu \mathrm{M})$ was included in cultures grown in either BME/EBSS or DMEM plus 10\% FBS for 3 days without streptomycin. After fixation, BPs were immunoreacted to detect BrdU.

BrdU-positive nuclei were detected in BPs grown in streptomycin-free cultures in DMEM (Fig. 5A, B) or BME/EBSS (image not shown) plus $10 \%$ FBS. We counted BrdU-positive nuclei in seven fields across the $\mathrm{BP}$, and we performed statistical analyses to assess the following features of these data: overall average BrdU labeling densities, widthwise gradients of average BrdU labeling densities, and lengthwise gradients of average BrdU labeling densities (see "Methods" for descriptions). In addition, we estimated the percentage of SCs that were BrdU-labeled. These data are presented in Table 2. Average BrdU labeling densities (nuclei per 
$10,000 \mu \mathrm{m}^{2}$ ) for each lengthwise site and widthwise region in each media type are graphed in Figure 5C.

We found no statistically significant difference between BPs cultured in DMEM and BPs cultured in BME/EBSS with respect to overall BrdU labeling densities (Table 2). Analysis of widthwise gradients (with proximal data omitted) showed that, in BPs grown in DMEM, average BrdU labeling densities were similar in neural and abneural halves of the BP, while in BPs grown in BME/EBSS, they were significantly higher in the abneural half. Analysis of lengthwise gradients revealed that, for both media, average BrdU labeling density was highest in the proximal end and lowest in the distal end. Based on the overall analysis, we estimated that approximately $2 \%$ of SCs cultured in either media type entered the cell cycle during the 3-day culture period in streptomycin-free conditions.

Supporting cell proliferation is upregulated after streptomycin treatment in vitro, with different responses in the two media types

Next, we examined rates of SC division in streptomycintreated cultures, in which complete $\mathrm{HC}$ loss was triggered. BrdU $(1 \mu \mathrm{M})$ and streptomycin $(78 \mu \mathrm{M})$ were added to DMEM plus 10\% FBS for 3 days. Cultures were fixed, and cumulative SC division was assessed by BrdU immunolabeling, similar to above. Averaged data are
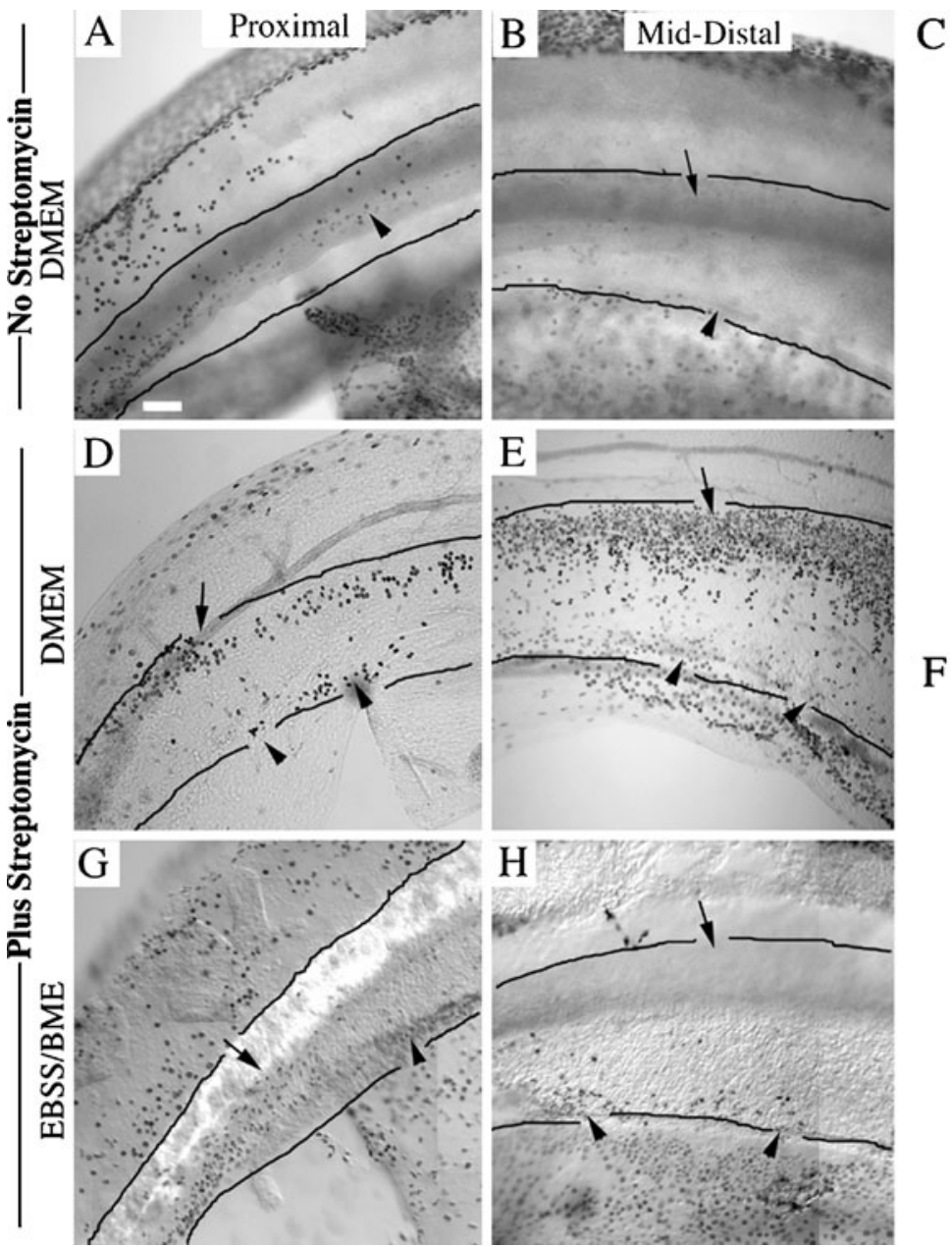

FIG. 5. Supporting cells divide in cultures with and without streptomycin. Digital images $(\mathbf{A}, \mathbf{B}, \mathbf{D}, \mathbf{E}, \mathbf{G}, \mathbf{H})$ show BrdU immunohistochemistry in different regions of the whole-mount BP after three continuous days of culture in DMEM/10\% FBS or BME/ EBSS $/ 10 \% \mathrm{FBS}$, with or without streptomycin (at $78 \mu \mathrm{M}$ ) and with BrdU $(1 \mu \mathrm{M})$ in the media. All images are focused on the sensory epithelium. The black lines demarcate the neural and abneural limits of the BP. In cultures maintained without streptomycin (A, B), several BrdU-positive nuclei (black dots) were present in the proximal end of the BP (A), but fewer BrdU-positive nuclei were present in the distal half (B). Average BrdU labeling densities for cultures grown without
$\mathrm{F}$

C
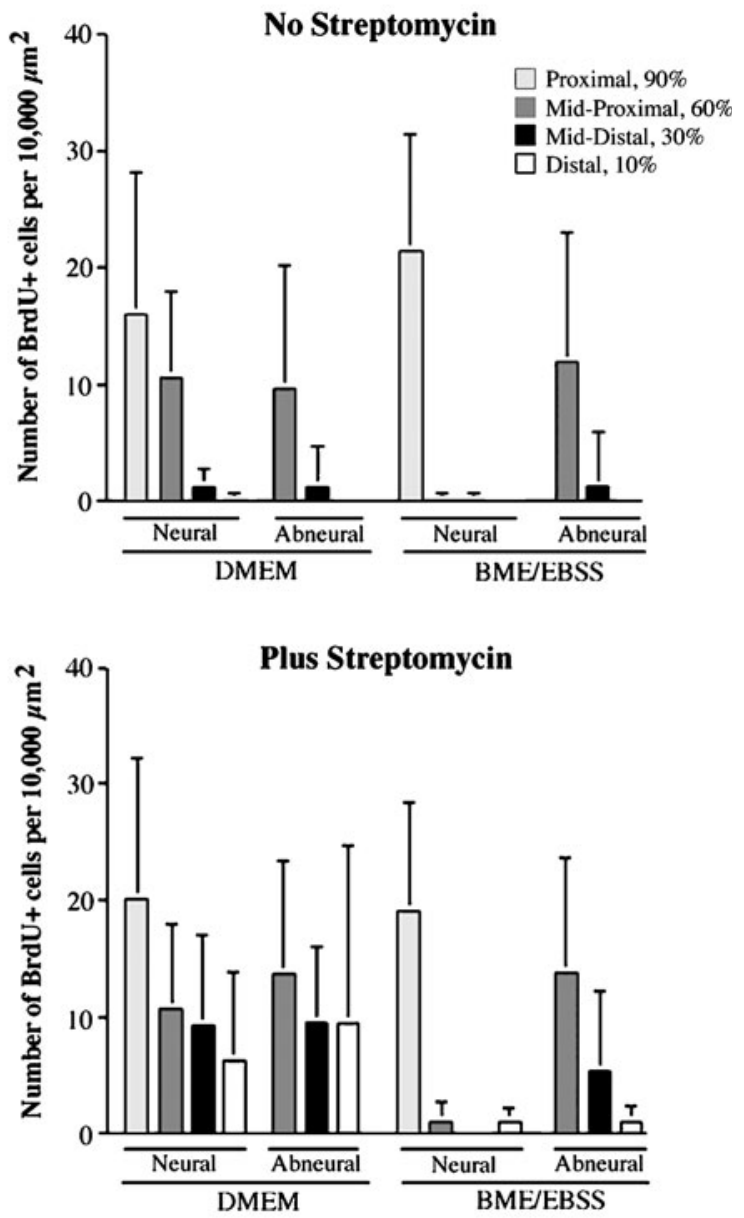

streptomycin in either DMEM or BME/EBSS (10\% FBS) are graphed in C (see Fig. 1A for sampling methods and Table 1 for Ns). Error bars represent SD. In cultures maintained in $\operatorname{DMEM}(\mathbf{D}, \mathbf{E})$ or BME/EBSS $(\mathbf{G}, \mathbf{H})$ plus $78 \mu \mathrm{M}$ streptomycin and $10 \% \mathrm{FBS}$, significantly more BrdU-positive nuclei were noted than in controls, particularly in middle and distal regions. BrdU labeling densities for streptomycintreated cultures are graphed in F. Error bars represent SD. In all images, proximal is toward the left, and abneural is down. Arrows point to the neural region of the $\mathrm{BP}$, and arrowheads point to the abneural region. Scale bar in $\mathbf{A}=$ in $50 \mu \mathrm{m}$ for $\mathbf{A}, \mathbf{B}, \mathbf{D}, \mathbf{E}, \mathbf{G}$, and $\mathbf{H}$. 
presented in Table 2, and average BrdU labeling densities for each lengthwise site and widthwise region in each media type are graphed in Figure 5F.

In contrast to streptomycin-free conditions, overall BrdU labeling densities varied according to culture media when streptomycin was present; BPs cultured in DMEM had statistically higher average BrdU labeling densities than BPs cultured in BME/EBSS (Table 2; compare Fig. 5D, E with Fig. 5G, H). Therefore, subsequent analyses of BrdU labeling for each media type are discussed independently.

For BPs cultured in DMEM, the overall BrdU labeling density was twofold higher in streptomycintreated BPs than in untreated BPs $(11.6 \pm 10.3$ versus $5.55 \pm 8.87$ labeled cells per $10,000 \mu \mathrm{m}^{2}, p<0.0001$; see Table 2; compare Fig. 5D, E with Fig. 5A, B). We estimated that $4.4 \%$ of SCs entered the cell cycle during the culture period.

Organs cultured in DMEM plus streptomycin had two areas of high BrdU labeling density that ran in stripes along the abneural and neural edges of the BP. These foci were easily distinguished in the middle and distal portions of the epithelium, but they were less evident in the proximal portion, which is thinner in width. This pattern was different from BPs cultured in streptomycin-free conditions, in which BrdU uptake was largely confined to SCs in the proximal region. The presence of two distinct foci of SC division in a large expanse of a lesion was surprising, since SC entry into the cell cycle generally mirrors HC loss (e.g., Stone and Cotanche 1994), and streptomycin-induced HC loss was widespread and not limited to just the neural and abneural edges. This was also surprising because no clear widthwise (neural-abneural) gradients were noted in the temporal progression of HC loss after streptomycin treatment. Statistical analysis of widthwise gradients showed that, in streptomycin-treated BPs grown in DMEM, the average BrdU labeling density in the neural region was not significantly different from the density in the abneural region (Table 2). Analysis of lengthwise gradients showed that average BrdU labeling densities varied significantly between the proximal site and all other sites $(p<0.05$; Table 2$)$, but they were similar between the distal and middistal sites $(p=0.605)$, the middistal and middle sites $(p=0.146)$, and the middle and midproximal sites $(p=0.344)$.

When we analyzed BPs cultured in BME/EBSS, we found no significant difference in the overall BrdU labeling densities between untreated BPs and streptomycin-treated BPs $(6.16 \pm 12.8$ versus $6.11 \pm 9.06$ labeled cells per $10,000 \mu \mathrm{m}^{2} ; p=0.977$; see Table 2; compare Fig. 5A, B with Fig. 5G, H). This observation was not anticipated, since streptomycin-treated BPs showed significantly more $\mathrm{HC}$ loss throughout the BP than streptomycin-free cultures. Analysis of widthwise gradients revealed that BrdU uptake in BPs cultured in BME/EBSS was mostly confined to SCs along the abneural edge (Table 2). This is in contrast to BPs cultured in DMEM, which had both neural and abneural foci of SC division. This observation was also surprising, since HCs had been lost throughout the entire BP.

Analysis of BPs cultured in BME/EBSS plus streptomycin showed significant variation in average BrdU labeling densities in different lengthwise sites, which was similar to that seen in BPs cultured in DMEM plus streptomycin (Table 2). For BME/EBSS, average BrdU labeling densities were significantly different between all sites $(p<0.05)$, except for between the distal and middistal sites $(p=0.392)$.

In drug-damaged cultures, higher serum levels are associated with decreased supporting cell division and altered organ growth

To determine effects of serum concentration on SC division, we compared cumulative BrdU labeling during 3 days of culture in DMEM or BME/EBSS containing $1 \%$, $5 \%$, or $10 \%$ FBS, BrdU $(1 \mu \mathrm{M})$, and streptomycin. We performed this analysis for control BPs and for BPs treated with streptomycin, and we determined average overall BrdU labeling densities for each serum concentration. BrdU-positive nuclei were detected using DAB immunohistochemistry, as shown in Fig. 5. Data are presented in Table 3.

In control cultures without streptomycin, there was no significant effect of serum concentration on overall BrdU labeling densities in either media type ( $p>0.2$ for all comparisons). Similarly, serum concentration had no effect on SC division in BPs cultured with streptomycin in BME/EBSS ( $p>0.3$ for all comparisons). In contrast, BrdU labeling densities tended to be inversely correlated with serum concentration in BPs cultured with streptomycin in DMEM. Differences were statistically significant between cultures grown in $1 \%$ and $10 \%(p=0.013)$ and cultures grown in 5\% and $10 \% \quad(p=0.014)$, but there was no significant difference between cultures grown in $1 \%$ and $5 \%$ FBS $(p=0.635)$. We observed that any cultures grown in $5 \%$ or $10 \%$ FBS had significant three-dimensional distortion, which included twisting of the organ into a spiral configuration. In contrast, cultures grown in 1\% FBS showed little or no distortion.

Collectively, these results indicate that low serum levels are beneficial for studies of hair cell regeneration following streptomycin treatment, due to increased SC division and reduced tissue distortion.

Supporting cell reentry into cell cycle after aminoglycoside treatment is similar in vitro and in vivo

In vivo, a few SCs in the BP enter S phase around 2 days after aminoglycoside treatment, and SC divi- 
sion peaks a day later (Bhave et al. 1995, 1998; Stone et al. 1999; Duncan et al. 2006). To determine if SCs enter $\mathrm{S}$ phase on a similar schedule after streptomycin-induced HC damage in vitro, $\operatorname{BrdU}(1 \mu \mathrm{M})$ was added to culture media (DMEM/1\% FBS) for $4 \mathrm{~h}$ at $1,2,3,5$, or 7 days in vitro, and cultures were fixed and immunolabeled for BrdU. For 1-, 2-, and 3-day cultures, streptomycin was present for the entire culture period. For 5- and 7-day cultures, streptomycin was present only for the first 2 days in culture.

No incorporation of BrdU into SC nuclei was seen at 1 day in vitro. In contrast, a significant number of SCs were BrdU-positive at 2 days (Fig. 6). At this time, the distribution of BrdU-positive SCs in neural and abneural regions of the BP was similar to that described in Figure 5 for tissue at 3 days in vitro (data not shown). Numbers of BrdU-positive SCs at 3 days resembled those at 2 days, but they were significantly decreased at later times. This time course is similar to what has been reported after aminoglycoside damage in vivo.

Hair cells are regenerated after streptomycin treatment. We next examined if auditory HCs are regenerated in vitro following drug damage. Organs were cultured with streptomycin for 2 days and then maintained for different periods afterward in streptomycin-free media. Cultures were fixed and immunolabeled to detect the HC marker, myosinVI. For these experiments and for the other experiments described below, organs were cultured in DMEM plus 1\% FBS, since these media supported a higher degree of SC division and caused less

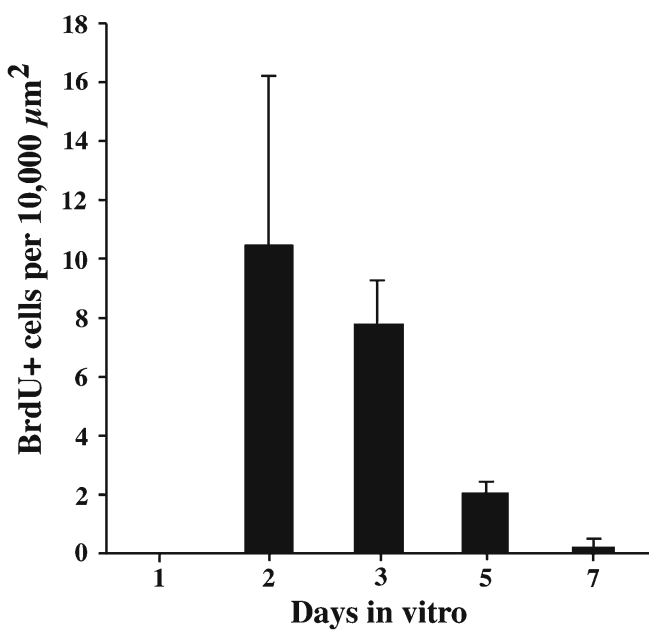

FIG. 6. Time course of supporting cell division after streptomycin treatment. Graph shows average overall BrdU labeling densities in whole-mounted cultures that were maintained in DMEM plus $10 \%$ FBS for different times after treatment with $78 \mu \mathrm{M}$ streptomycin. Sampling methods are depicted in Figure 1A. Error bars represent SD. ANOVAs showed that densities at 2 and 3 days were significantly different $(p<0.05)$ from all of the other times, but they were not different from each other. Also, average densities in cultures at 1, 5, and 7 days were not statistically different from each other $(\geq 0.05)$. three-dimensional organ distortion than higher serum concentrations.

As described above, BPs cultured for 2 days plus streptomycin followed by 1 day without streptomycin had very few surviving original HCs (Fig. 7A). In BPs cultured for 8 days ( 2 days plus streptomycin followed by 6 days without streptomycin), myosinVI-positive cells had reemerged in the BP and were scattered throughout the epithelium in a relatively even pattern (Fig. 7B). Many such cells had either fusiform cell shapes, resembling immature regenerated HCs in vivo (e.g., Stone and Rubel 2000), or rounded cell shapes, resembling more mature HCs. Immunolabeling for additional HC markers-class III B-tubulin and calmodulin (Stone and Rubel 2000) - also revealed the return of HC-like cells in streptomycin-treated cultures (data not shown). On average, BPs that were dissected from control chicks and immediately fixed had 83.2 $( \pm 5.42)$ myosinVI-positive cells per $10,000 \mu \mathrm{m}^{2}$ (see Fig. 4A) compared to $10.5( \pm 1.27)$ myosinVI-positive cells per $10,000 \mu \mathrm{m}^{2}$ in BPs that were cultured for 8 days (with streptomycin for the first 2 days). Sampling methods for these counts are depicted in Figure 1B. Thus, the density of regenerated HCs in BPs cultured for 8 days (with streptomycin for the first 2 days) was about $13 \%$ that of uncultured control BPs. Maintenance of BPs for a longer time (12 days total, with the first 2 days containing streptomycin) resulted in an apparent increase in the density of myosinVIpositive cells compared to 8-day cultures (Fig. 7C). For comparison, Figure 7D shows a BP dissected from a chicken 10 days after in vivo gentamicin treatment and fixed immediately.

Examination of BPs after shorter culture periods showed that myosinVI-positive HCs were first detectable at 4 days in vitro (2 days plus streptomycin followed by 2 days minus streptomycin; Fig. 7E). New HCs initially appeared in the middle of the BP (lengthwise) in a similar region to where $\mathrm{HC}$ extrusion was first noted (Fig. 2). Such cells resembled early regenerated HCs in vivo between 3 and 4 days postgentamicin (Stone and Rubel 2000; Mangiardi et al. 2004; Roberson et al. 2004). They had very immature morphologies, with nuclei positioned in the SC nuclear layer and long constricted necks that extended from the nucleus toward the lumenal surface. In similar cultures in which $1 \mu \mathrm{M}$ BrdU was added from the start, some myosinVI-positive cells (presumed immature HCs) were BrdU-positive, indicating they had been formed by cell division (Fig. 7F). Other presumed regenerated HCs had no detectable BrdU labeling. Examination of BPs cultured for 8 days ( 2 days plus streptomycin followed by 6 days without streptomycin, with BrdU always present) revealed that $13 \%( \pm 7 \%)$ of myosinVI-positive cells were BrdU positive and $87 \%( \pm 7 \%)$ were BrdU negative (Fig. $7 \mathrm{G}, \mathrm{H}$ ). This observation suggested that most HCs regenerated in 
Plus Strept, in vitro
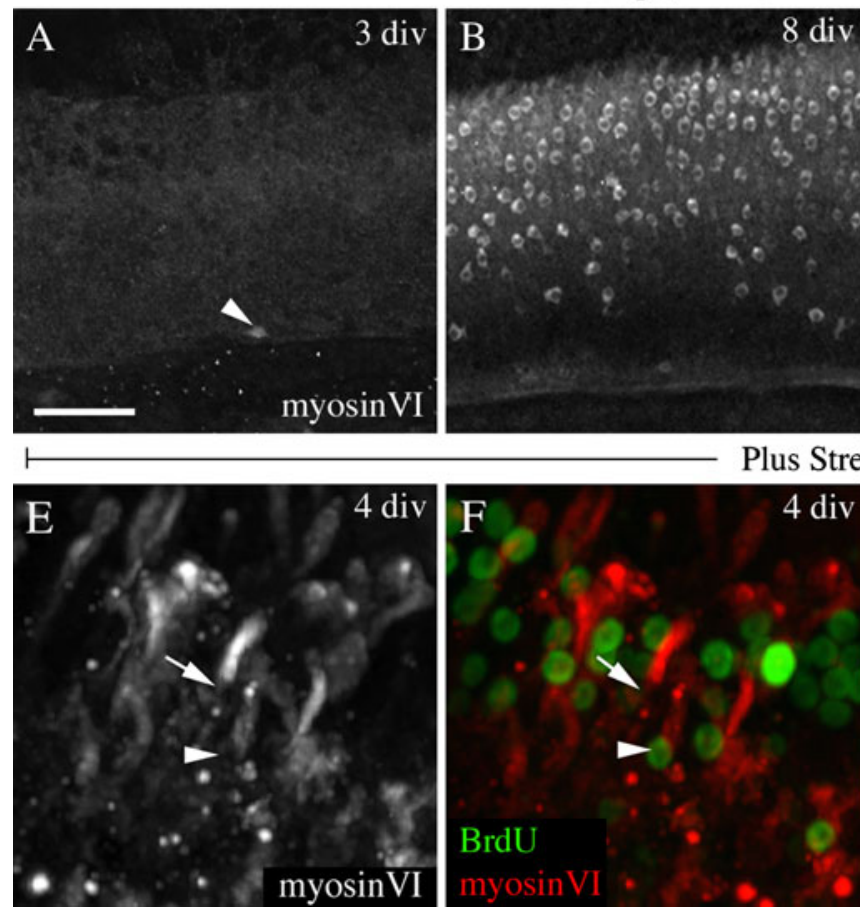

Plus Strept, in vitro

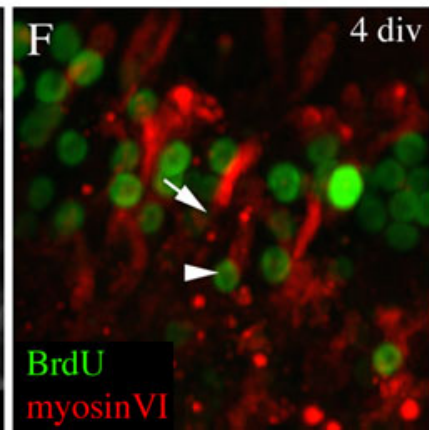

FIG. 7. Hair cells are regenerated in culture. Panels A-C show myosin VI immunolabeling in the midproximal region of wholemount BPs that were cultured in DMEM and 1\% FBS for 2 days with $78 \mu \mathrm{M}$ streptomycin (Strept) followed by periods in the same media without streptomycin, for a total of 3 days (A), 8 days (B), or 12 days $(\mathbf{C})$ in vitro. The arrow in $\mathbf{A}$ indicates an original $\mathrm{HC}$ that remained in the epithelium after streptomycin treatment. Panel D shows myosinVI labeling in a similar region at 10 days after gentamicin (Gent) treatment in vivo. In A-D, proximal is toward the left, and abneural is downward. Panels $\mathbf{E}$ and $\mathbf{F}$ are images taken from one field located in the middle of a BP after 4 days of culture (including an initial 2 days of streptomycin). MyosinVI labeling is shown in $\mathbf{E}$, and both myosinVI (cytoplasmic label) and BrdU (nuclear label) labeling are

culture were formed by direct transdifferentiation of SCs rather than by SC mitosis. This interpretation was further explored (described below). We found that $5 \%$ of BrdUpositive cells were myosinVI-positive, indicating that, at 8 days in culture, only a small percentage of postmitotic cells had begun to differentiate as HCs.

To explore the extent to which HC features differentiate in culture, we counter-labeled 8-day cultures with antibodies to hair cell antigen (HCA). HCA is a glycoprotein present in developing and mature stereociliary bundles of avian HCs (Bartolami et al. 1991). It is expressed at a later stage of differentiation during in vivo regeneration than myosinVI (Stone and Rubel 2000). HCA labeling was evident atop some myosinVIpositive/BrdU-negative cells (Fig. $7 \mathrm{H})$, but it was detected rarely on myosinVI-positive/BrdU-positive cells. Of 426 myosinVI-positive cells examined throughout three BPs, apical HCA labeling was detected in 365 BrdU-negative cells, but it was detected in only one BrdU-positive cell.

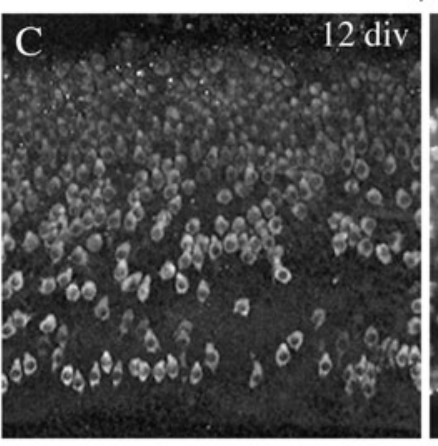

Plus Gent, in vivo
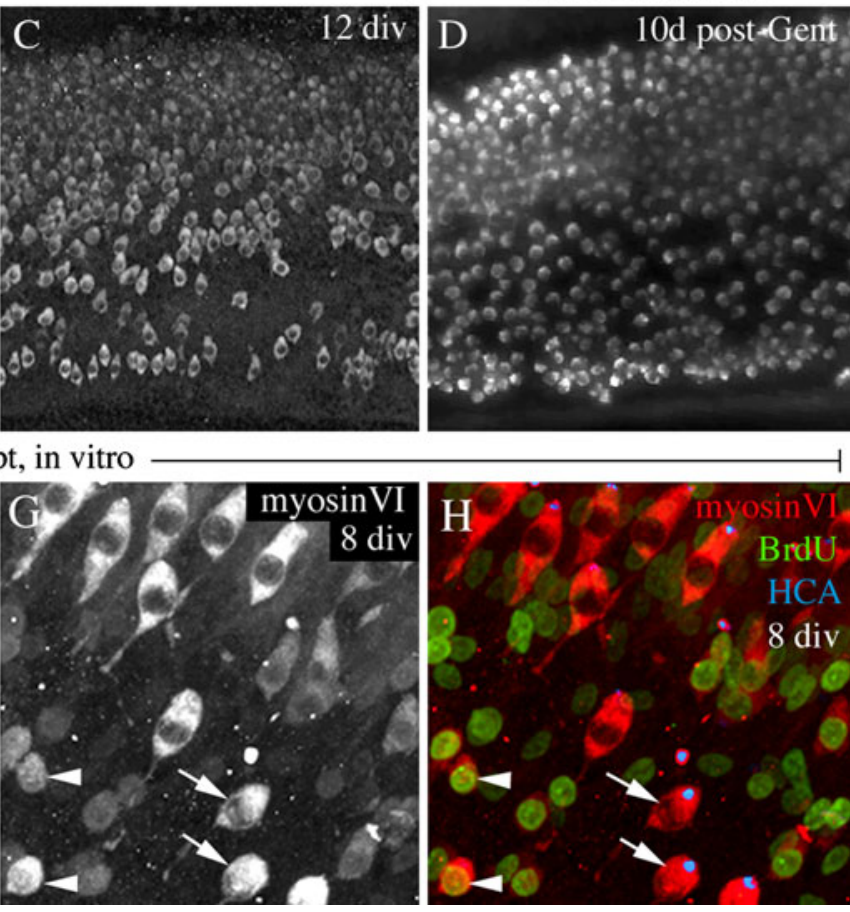

shown in $\mathbf{F}$. The nuclei of two regenerated HCs are indicated by an arrow and an arrowhead in each panel. In both cells, long myosinVIpositive necks extend upward, toward the lumen. The arrow points to a cell that has a BrdU-negative nucleus; the arrowhead points to a cell with a BrdU-positive nucleus. Panels $\mathbf{G}$ and $\mathbf{H}$ are images taken from one field in the middle of the BP after 8 days of culture, with myosin VI labeling in $\mathbf{G}$ and with myosinVI (cytoplasmic label) and BrdU (nuclear label) labeling shown in $\mathbf{H}$. Arrowheads point to myosinVI-positive cells that are BrdU-positive, while arrows point to myosinVl-positive cells that are BrdU-negative and also have HCApositive bundles (small dots) atop them. Scale bar in $\mathbf{A}=50 \mu \mathrm{m}$ for $\mathbf{A}-$ $\mathbf{D}$ and $15 \mu \mathrm{m}$ for $\mathbf{E}-\mathbf{H}$.

In a few experiments, we examined $\mathrm{HC}$ regeneration in BPs cultured in BME/EBSS with 1\% FBS for 2 days plus streptomycin followed by 6 days without streptomycin. We noted that HCs were also regenerated under these conditions, but further analyses were not performed.

\section{Hair cells are regenerated in serum-free media}

To investigate if $\mathrm{HC}$ regeneration occurs in the absence of serum, we cultured organs in DMEM plus streptomycin and $1 \mu \mathrm{M}$ BrdU for 3 days, with no FBS present at any time. Analysis of BrdU immunohistochemical labeling showed that SCs continued to enter S phase in the absence of serum, in a spatial pattern resembling that seen in organs cultured with 1-10\% FBS present (Fig. 8A, compare with Fig. 5D, E). There was no statistically significant difference in the overall BrdU labeling densities of BPs grown without FBS compared to BPs grown with $1 \%$ or $5 \%$ FBS (Table 3). However, BPs cultured without FBS had significantly higher overall 
BrdU labeling densities compared to BPs cultured with $10 \%$ FBS. Mitotic figures were evident in the sensory epithelium (Fig. 8A, inset), indicating that SCs were able to complete the cell cycle in the absence of serum. In addition, myosinVI-positive cells that were either BrdUpositive (Fig. 8B) or BrdU-negative (Fig. 8C) reemerged in serum-free cultures maintained for 2 days with streptomycin followed by 4 days without streptomycin (with BrdU present the entire time), indicating that both nonmitotic and mitotic $\mathrm{HC}$ regeneration had occurred.

\section{Hair cell differentiation is inhibited by BrdU}

In other tissues, BrdU can hamper cellular differentiation, due to its inhibition of transcription of certain genes (Levitt and Dorfman 1972; Tapscott et al. 1989). To test if BrdU concentration affects differentiation of regenerated HCs in vitro, we determined

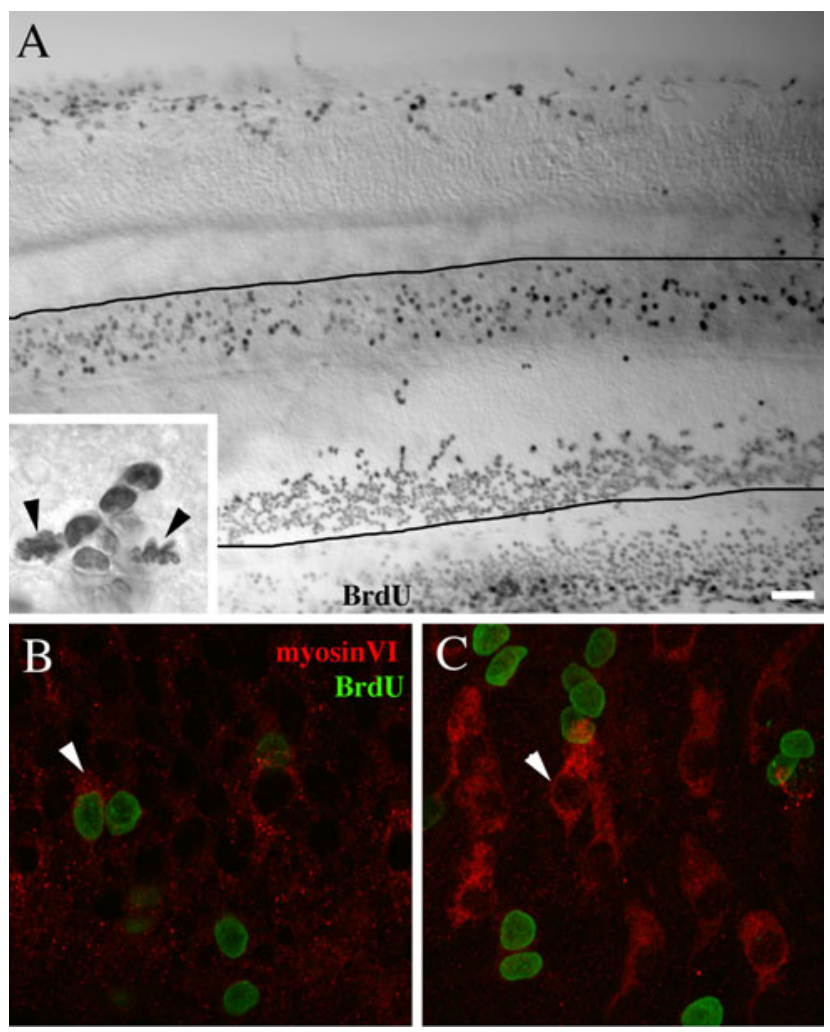

FIG. 8. Supporting cell division and hair cell differentiation proceed in serum-free conditions. All panels show BPs cultured in serum-free DMEM and $1 \mu \mathrm{M} \mathrm{BrdU}$ for the whole culture period. A This panel shows $\mathrm{BrdU}$ labeling in the middle region of a BP cultured for 3 days with $78 \mu \mathrm{M}$ streptomycin prior to fixation. Distal is toward the right, and abneural is downward. The edges of the BP are demarcated by black lines. In the inset, BrdU-positive cells are shown at higher magnification, including two mitotic figures (arrows). B, C MyosinVI and BrdU labeling in a BP cultured for 2 days with $78 \mu \mathrm{M}$ streptomycin followed by 4 days without streptomycin. Arrowhead in B points to a cell that is myosinVI positive and BrdU positive; arrowhead in $\mathbf{C}$ points to a cell that is myosinVI positive and BrdU negative. Scale bar in $\mathbf{A}=30 \mu \mathrm{m}$ for $\mathbf{A}, 5 \mu \mathrm{m}$ for inset in $\mathbf{A}$ and for $\mathbf{B}$ and $\mathbf{C}$. the average density of regenerated myosinVI-positive cells in 8-day cultures (2 days plus streptomycin then 6 days without streptomycin) grown with either $0,0.1$, or $1 \mu \mathrm{M}$ BrdU for the entire culture period.

We found no statistically significant difference in myosinVI labeling densities between BPs cultured with 0 or $0.1 \mu \mathrm{M}$ BrdU, but there was a significant decrease in myosinVI labeling densities in BPs cultured in $1 \mu \mathrm{M}$ compared to the other two groups $(p<0.05$; Fig. 9). These findings suggested that the higher concentration of BrdU either prevented or delayed differentiation of regenerated HCs, as measured by myosinVI immunoreactivity. BPs cultured with $1 \mu \mathrm{M}$ BrdU had statistically higher average BrdU labeling densities than BPs cultured with $0.10 \mu \mathrm{M} \operatorname{BrdU}(p<0.05$; Fig. 9). This observation most likely reflects the need for a minimum nuclear accumulation of $\mathrm{BrdU}$ for its detection via immunofluorescence. These findings serve as an important reminder that estimates of SC proliferation and HC differentiation are largely influenced by the amount of BrdU present in culture media.

Supporting cell division is drastically inhibited with aphidicolin treatment

Our observation that a large proportion of HCs formed in culture lacked nuclear BrdU labeling suggested that direct transdifferentiation (i.e., conversion of SCs into HCs without cell division) is a major mode of new HC production in chicken BPs in vitro. If this were true, then large numbers of HCs should be regenerated, even when SC division is inhibited. To address this, we examined the ability

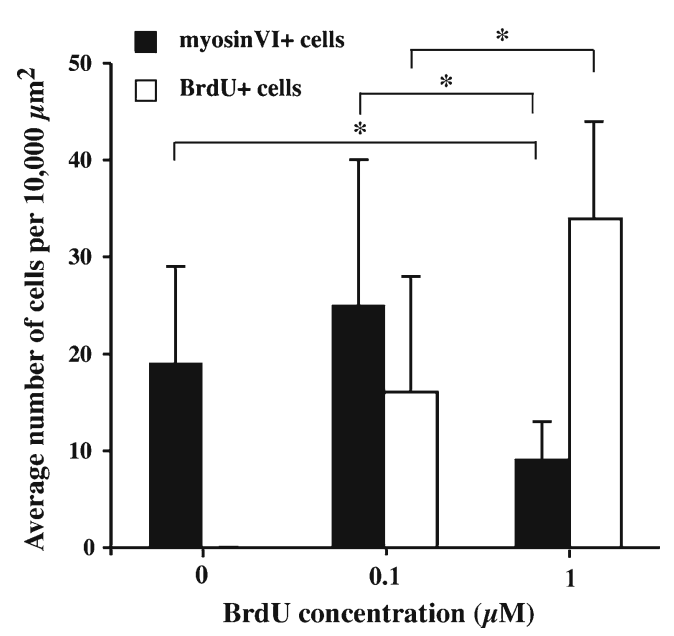

FIG. 9. BrdU inhibits differentiation of hair cells. The average density of BrdU-positive cells (white bars) or myosinVI-positive cells (black bars) is shown for 8 -day cultures grown in DMEM plus $1 \%$ FBS for 2 days with $78 \mu \mathrm{M}$ streptomycin followed by 6 days in the same media without streptomycin, with different concentrations of $\mathrm{BrdU}$ present. The sampling method for these counts is depicted in Figure 1C. Asterisks signify significant differences between indicated groups $(p \leq 0.05$, ANOVA with Fisher's PLSD). Error bars represent SD. 
of the DNA polymerase inhibitor, aphidicolin, to prevent SC division. Aphidicolin blocks a wide range of cell types from progressing past the G1/S border of the cell cycle (Pedrali-Noy et al., 1980). At $25 \mu \mathrm{M}$, aphidicolin has also been shown to block SC division in newt saccules (Taylor and Forge 2005) and frog saccules (Baird et al. 1996, 2000; Gale et al. 2002).

In pilot experiments, we tested whether aphidicolin has noticeable toxic effects on mature HCs by culturing organs for 3 days with $25 \mu \mathrm{M}$ aphidicolin (dissolved in $1 \%$ DMSO) or $1 \%$ DMSO without streptomycin. After culture, BPs were labeled to detect myosinVI. We found no qualitative difference in the density or appearance of myosinVI-positive cells between the two culture conditions (data not shown), suggesting that aphidicolin itself is not toxic to HCs.

To test if aphidicolin prevents SCs from dividing, organs were dissected and cultured for 3 days with streptomycin and either $1 \%$ DMSO or $25 \mu \mathrm{M}$ aphidicolin. For the last day in culture, $1 \mu \mathrm{M}$ BrdU was included to label dividing cells. Media were replenished daily. Organs were fixed and immunolabeled to detect BrdU. In DMSO-treated BPs, nuclei with strong BrdU labeling were abundant and appeared small and round (Fig. 10A), similar to cultures described in Figure 5. Some BrdU-positive mitotic figures were evident (Fig. 10A, inset). In contrast, BrdU-positive nuclei were rare in aphidicolin-treated BPs, and they appeared very different than in DMSO-treated cultures; they were larger in size and much more lightly labeled with BrdU (Fig. 10B). Similar observations were made in the cartilage for each group of BPs (see insets Fig. 10A, B). Further, we found that treatments of aphidicolin as short as $6 \mathrm{~h}$ prevented significant BrdU uptake (data not shown).

The paucity of BrdU-labeled nuclei in aphidicolintreated BPs suggested that, at least during the third day in culture, many SCs were unable to progress into $\mathrm{S}$ phase. To test if SCs that did make it to $\mathrm{S}$ phase were able to complete the cell cycle, we immunolabeled BPs in each group for $\mathrm{pH} 3$, a marker specific for cells in mitosis, and counter-labeled them with propidium iodide, a dye for nucleic acids (Fig. 10C, D). The entire BP was scanned using confocal microscopy for pH3-positive cells. On average, each DMSO-treated $\mathrm{BP}$ had 28.8 ( \pm 6.80) pH3-positive cells, but each aphidicolin-treated BP lacked pH3-positive cells, confirming that $25 \mu \mathrm{M}$ aphidicolin prevented all SCs from reaching mitosis and dividing. We performed similar experiments with aphidicolin at lower doses $(2.5$ and $10 \mu \mathrm{M})$, but we found they permitted significantly more SC division (data not shown). Therefore, these lower doses were not used in subsequent experiments.
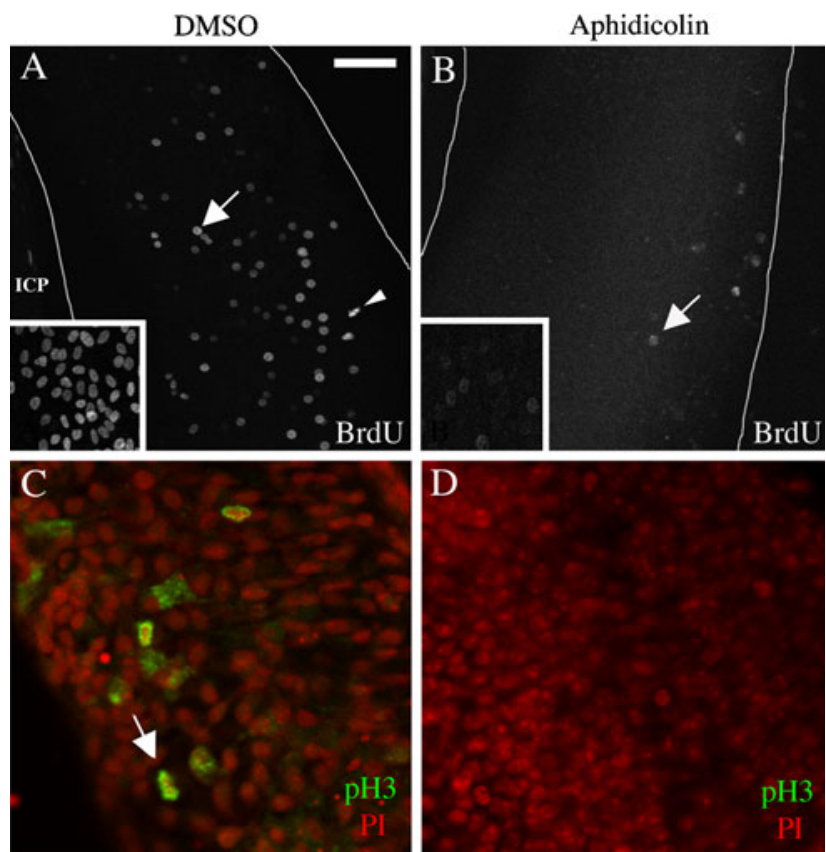

FIG. 10. Aphidicolin prevents supporting cell division. All panels show whole-mounted BPs cultured for 3 days in DMEM plus $1 \%$ FBS and $78 \mu \mathrm{M}$ streptomycin, supplemented with either $1 \%$ DMSO $(\mathbf{A}, \mathbf{C})$ or $25 \mu \mathrm{M}$ aphidicolin $(\mathbf{B}, \mathbf{D})$. Thin white lines in $\mathbf{A}$ and $\mathbf{B}$ show the neural and abneural borders of the BP (neural is toward the right). The midproximal region is shown in all panels. In $\mathbf{A}$, a portion of the inferior cartilage plate $(I C P)$ covers the abneural edge of the BP. A DMSO controls had numerous BrdU-positive cells in the BP and the middle of the ICP (inset). BrdU labeling in interphase nuclei (arrow) and in mitotic chromatin (arrowhead) was evident. B Aphidicolintreated BPs showed dramatic reduction in the number of BrdUpositive cells and the intensity of BrdU labeling, in both the BP (arrow) and the ICP (inset). DMSO-treated BPs had numerous pH3positive mitotic figures (C), while aphidicolin-treated BPs (D) had none. Propidium iodide $(P I)$ labeling (nuclear label in $\mathbf{C}$ and $\mathbf{D}$ ) indicates location of all nuclei. Scale bar in $\mathbf{A}=30 \mu \mathrm{m}$ for $\mathbf{A}$ and $\mathbf{B}$ (and insets) and $15 \mu \mathrm{m}$ for $\mathbf{C}$ and $\mathbf{D}$.

Hair cells continue to be regenerated despite blockade of supporting cell division

Next, we examined if HCs are regenerated when SC division is drastically attenuated. Organs were cultured in streptomycin for 2 days and in streptomycinfree media for six additional days. In half of the cultures, $25 \mu \mathrm{M}$ aphidicolin was added to media for the whole culture period. The remaining cultures were treated for the whole culture period with $1 \%$ DMSO or with nothing (controls). BrdU $(1 \mu \mathrm{M})$ was included for the entire culture period to assess cumulative SC division. Media were replenished daily. Cultures were fixed and triple-labeled to detect myosinVI, HCA, and BrdU.

Numerous BrdU-positive nuclei were detected in BPs cultured in control media (Fig. 11A) or with DMSO (Fig. 11B). In both cases, BrdU-labeled nuclei were present in myosinVI-positive cells (presumed regener- 

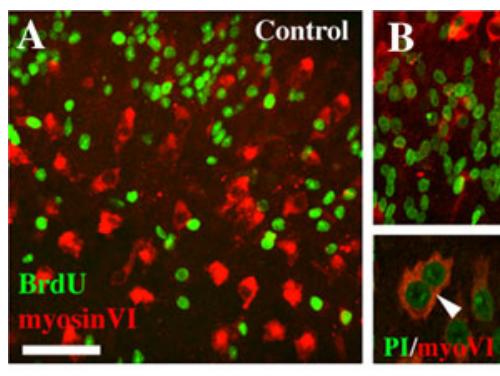

D
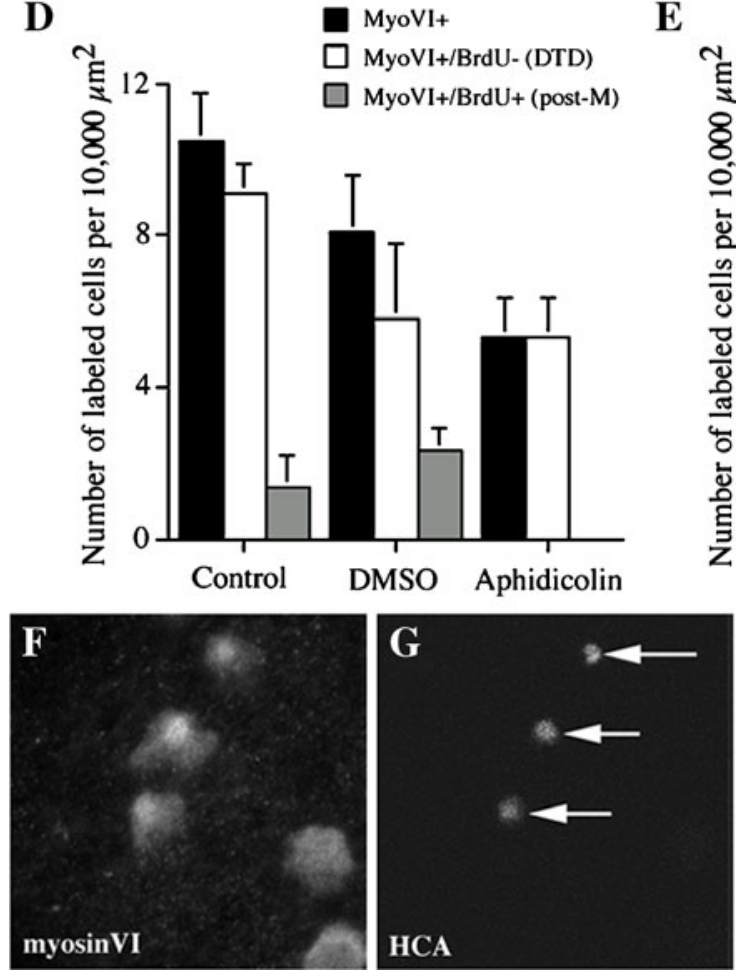

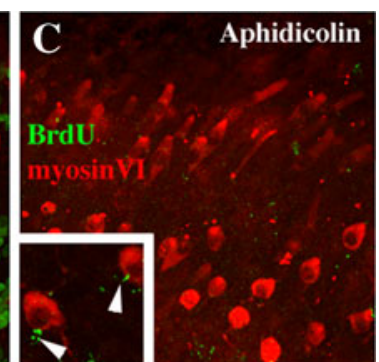

E
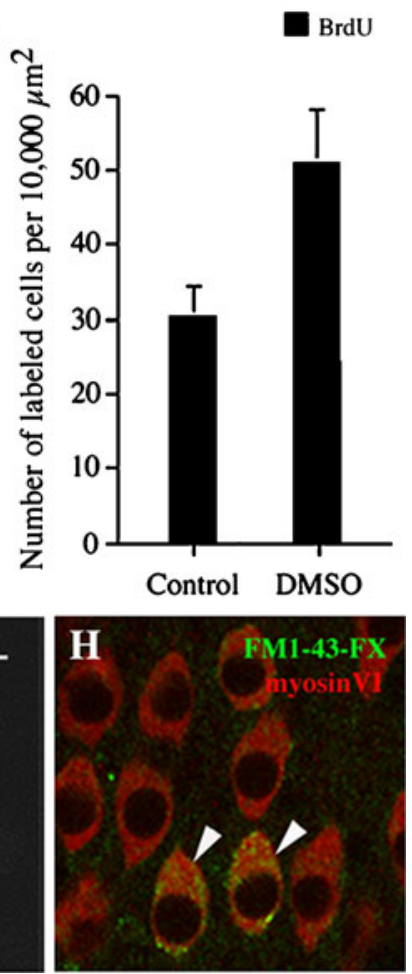

FIG. 11. Regeneration of differentiated hair cells proceeds when supporting cell division is blocked. A-C show BPs cultured for 8 days (2 days plus $78 \mu \mathrm{M}$ streptomycin followed by 6 days without streptomycin) in control media (DMEM/ $1 \%$ FBS; A), media plus 1\% DMSO (B), or media plus $25 \mu \mathrm{M}$ aphidicolin (C). BPs were immunolabeled for myosinVI (cytoplasmic label) and BrdU (nuclear label). The arrowhead in $\mathbf{B}$ inset points to two fused myosinVI-positive cells with propidium-iodide $(P I)$-labeled nuclei. The inset in $\mathbf{C}$ shows BrdU-positive cell fragments (arrowheads). Average labeling densities for myosinVI-positive cells for each treatment group cultures are graphed in $\mathbf{D}$. MyoVI myosinVI, DTD direct transdifferentiation, Post-M postmitotic. Average labeling densities for BrdU-positive cells for each treatment group are graphed in E. The sampling method for counts in D and $\mathbf{E}$ is depicted in Figure 1B. Error bars in both graphs represent SD. F and $\mathbf{G}$ show the same field from a 10-day culture, double-labeled for myosinVI and HCA. Arrows in G point to HCA-positive stereociliary bundles atop myosinVI-positive cells in $\mathbf{F}$. $\mathbf{H}$ shows a 12-day culture doublelabeled for myosinVI (label throughout cytoplasm) and FM1-43-FX (punctate label in cytoplasm). Arrowheads point to two double-labeled cells. Scale bar in $\mathbf{A}=25 \mu \mathrm{m}$ for $\mathbf{A}-\mathbf{C}, 15 \mu \mathrm{m}$ for $\mathbf{B}$ and $\mathbf{C}$ insets, and $10 \mu \mathrm{m}$ for $\mathbf{F}-\mathbf{H}$. ated HCs) as well as in myosinVI-negative cells (presumed regenerated SCs). In contrast, no BrdU-positive nuclei were seen in aphidicolin-treated BPs (Fig. 11C), although tiny fragments of BrdU labeling were sometimes evident (Fig. 11C, inset). Numbers of BrdUpositive nuclei were also drastically reduced in tissues outside the SE, such as the cartilage (data not shown).

Despite the lack of BrdU-positive SCs in aphidicolin-treated BPs, a significant number of myosinVIpositive cells had reemerged in all regions (Fig. 11C). These cells had morphologies resembling regenerated HCs in control and DMSO-treated cultures. Similar results were seen with antibodies to the HCselective protein, calmodulin (data not shown). We noted that some regenerated HCs in cultures that included DMSO appeared to be fused (Fig. 11B, inset); such cells were not noted in control cultures.

Counts of myosinVI-labeled and/or BrdU-labeled cells in the three culture groups are shown in Figure 11D. MyosinVI labeling densities were significantly different between all three groups $(p<0.05)$, with controls having the highest densities and aphidicolin- treated BPs having the lowest. Densities of new HCs formed without SC division (myosinVI-positive/BrdUnegative) were significantly higher in controls than in DMSO-treated cultures $(p=0.009)$ or aphidicolintreated cultures $(p=0.003)$, although there was no significant difference between DMSO- and aphidicolin-treated cultures $(p=0.656)$. DMSO-treated BPs had significantly more BrdU-positive nuclei than controls $(p=0.000$; Fig. 11E). However, the density of myosinVI-positive/BrdU-positive cells was not significantly different between controls and DMSOtreated BPs $(p=0.075)$, suggesting that DMSO did not affect the rate of differentiation of HC-like features among postmitotic cells.

We examined whether myosinVI-positive cells regenerated in the absence of SC division are able to differentiate advanced HC features, including stereociliary bundles and functional mechanotransduction channels, if maintained for longer periods in culture. Organs were cultured for either 10 days (2 days plus streptomycin followed by 8 days without streptomycin) or 12 days ( 2 days plus streptomycin followed by 10 days 
without streptomycin), with $25 \mu \mathrm{M}$ aphidicolin and $1 \mu \mathrm{M}$ BrdU present for the entire culture period. BPs were fixed and immunoreacted to detect the stereocilia marker HCA, or they were live-labeled with FM1-43 for a very short period. This latter measure has been shown to reflect differentiation of mechanotransduction by HCs in other studies (Géléoc and Holt 2003; Si et al. 2003). In 10-day cultures treated with aphidicolin, many myosinVI-positive cells had begun to develop bundles of stereocilia, as reflected by colabeling for HCA (Bartolami et al. 1991; Fig. 11E, F). In 12-day cultures, 39\% $( \pm 10 \%)$ of myosinVI-positive cells also showed significant uptake of FM1-43-FX (Fig. 11G), suggesting they had operational mechanotransduction channels.

The finding that HCs are regenerated in the absence of SC division strongly suggests that SCs are able to directly convert into HCs (i.e., to undergo direct transdifferentiation). If this were true, we should be able to detect cells in the process of conversion that possess features of both SCs and $\mathrm{HCs}$, as has been demonstrated in the chicken BP after in vivo HC damage (Cafaro et al. 2007). To test this hypothesis, we labeled BPs that were cultured with streptomycin for 3 days with antibodies to Sox2 or Btectorin precursor, both of which are selective markers for SCs (Goodyear et al. 1996; Cafaro et al. 2007; Daudet et al. 2009), and antibodies to Atoh1, an early marker of differentiating HCs (Cafaro et al. 2007). In these cultures, we indeed found examples of cells that labeled for Atoh1 and either Sox2 or Btectorin precursor, suggestive of cells transferring from an SC to an HC state (Supplementary Fig. 2).

Collectively, our results demonstrate that a significant number of new HCs regenerated in vitro are formed by direct transdifferentiation, a nonmitotic process. They also show that HCs formed via direct transdifferentiation could be functional, since they develop stereocilia and they rapidly incorporate FM1-43.

\section{Epithelial cells are reduced in number} after aphidicolin treatment

Treatment with aphidicolin in cultures incubated with streptomycin should result in depletion of SCs because (1) it prevents mitotic replacement of SCs that directly transdifferentiate into HCs and (2) some SCs that enter $\mathrm{S}$ phase may undergo cell death. To test whether SCs are in fact depleted in aphidicolintreated BPs compared to DMSO controls, organs were cultured with streptomycin for 2 days followed by 6 days without streptomycin. Organs were labeled for the nuclear dye, propidium iodide, and the density of nuclei in the middle region of the $\mathrm{BP}$ was calculated.

On average, BPs treated with $1 \%$ DMSO had $39.6 \pm$ 3.98 nuclei per $10,000 \mu \mathrm{m}^{3}$, and BPs treated with $25 \mu \mathrm{M}$ aphidicolin had $28.5 \pm 2.38$ nuclei per $10,000 \mu \mathrm{m}^{3}$ $(p<0.001)$. This represents an approximate $28 \%$ decrease in HC and/or SC density in aphidicolintreated BPs compared to DMSO controls. Based on this analysis alone, we are unable to state whether this decrease represented fewer HCs, SCs, or both.

\section{Aphidicolin-treated basilar papillae lack evidence} for cell death

Our finding that a few BrdU-labeled SCs were present after 3 days of treatment with aphidicolin but absent after 8 days of treatment suggests that some cells that enter the cell cycle in the presence of aphidicolin may die and get extruded from the epithelium. To address this, organs were cultured with streptomycin for 3 days and were treated with $25 \mu \mathrm{M}$ aphidicolin for either 6 or $24 \mathrm{~h}$, with $1 \mu \mathrm{M}$ BrdU present. Tissue was fixed and reacted to detect the TUNEL reaction, a marker of dying cells. We found no evidence for increased TUNEL-positive cells in aphidicolin-treated BPs relative to controls (data not shown), although it is possible that cell death occurred in a more rapid or delayed manner than for which we tested.

\section{DISCUSSION}

\section{Patterns of $\mathrm{HC}$ loss vary in control and streptomycin-treated cultures}

Hair cells are highly susceptible to oxidative stress and to ototoxic agents, such as aminoglycoside antibiotics like streptomycin. In control cultures lacking streptomycin, substantial extrusion of HCs from the proximal $\mathrm{BP}$ was evident by 2 days in vitro; after this, HC loss spread toward the middle region. This was true for organs cultured in DMEM or BME/EBSS. Similar results were reported by Frenz et al. (1998) using medium 199 and by Oesterle et al. (1993) and Cheng et al. (2003) using BME/EBSS. Spontaneous HC loss was likely caused by the explantation process or deficiencies in culture conditions. HC death might be prevented by inhibiting molecules in the apoptotic pathway, such as caspases, as has been accomplished in cultures of whole utricles from chickens (Matsui et al. 2002) and mice (Cunningham et al. 2002).

In cultures treated with streptomycin, clear evidence for HC loss was detected a day earlier than in control cultures. Acceleration of HC loss in cultured chicken BPs by aminoglycosides has been described in other studies (Frenz et al. 1998; Cheng et al. 2003). With 2-day streptomycin treatments, we first detected HC loss in the middle BP (halfway between the proximal and distal tip), and it spread proximally and distally over time, until complete HC loss was seen around 3 days in vitro. This pattern was distinct from the proximal-to-distal gradient in HC loss seen 
in our control cultures or described after shorter in vitro exposures with neomycin or gentamicin (Frenz et al. 1998; Cheng et al. 2003). A proximal-to-distal gradient more closely resembles what is seen in vivo after systemic treatment with aminoglycosides, such as gentamicin (e.g., Hashino et al. 1991; Janas et al. 1995). Experiments using brief (2-h) exposures demonstrated that these differences were not specific to streptomycin. Rather, the odd pattern of HC loss seen with 2-day exposures would appear to reflect a dysregulation of normal ototoxicity mechanisms due to the prolonged streptomycin treatment. Extended exposure to streptomycin may offset normal drug uptake mechanisms, and additional factors associated with culture conditions may bias middle HCs toward earlier extrusion. Despite these differences, a pattern of complete HC loss has significant advantages for molecular analyses that require large amounts of uniform starting tissue. It has additional advantages for studies addressing $\mathrm{HC}$ regeneration in general, since it eliminates the possibility that repair of injured HCs could account for a return in HC number, which has been raised in other studies of drug-treated inner ear epithelia (e.g., Gale et al. 2002).

\section{Patterns of SC proliferation vary in control and streptomycin-treated cultures}

In the posthatch avian $\mathrm{BP}$, the $\mathrm{SC}$ population is normally quiescent but reenters the cell cycle following HC damage (Ryals and Rubel 1988; Corwin and Cotanche 1988; Raphael 1992; Hashino and Salvi 1993; Stone and Cotanche 1994). Subsequently, postmitotic cells differentiate into either HCs or SCs. Previous studies demonstrated that SCs from the chicken BP reenter the cell cycle in organ cultures (Oesterle et al. 1993; Navaratnam et al. 1996; Warchol and Corwin 1996) and in pure SC cultures (Stone et al. 1996) following HC loss. In this study, the distribution of dividing SCs was different in streptomycin-treated cultures than in control cultures. In control cultures, SC division was largely confined to the proximal end of the BP, while in streptomycin-treated cultures, SCs divided throughout the BP. Further, significantly fewer dividing SCs were seen in control cultures than in drugtreated cultures. These differences generally reflected the different patterns and degrees of HC loss seen in control and drug-damaged organs. However, dividing SCs were focused in two stripes - along the neural and abneural edges - that did not reflect the widespread loss of HCs in a given region. A neural stripe of SC division was described for the chicken BP after in vivo gentamicin treatment (Cafaro et al. 2007), although the significance of this pattern is not understood. It is unclear if the abneural stripe of SC division is a culture artifact or a normal phenomenon that is amplified by culture conditions. This pattern was similar in cultures grown in the absence of serum, suggesting that it is not controlled by components present in serum.

Culture media also influenced the pattern of SC division in both control and drug-treated cultures. BPs cultured in BME/EBSS consistently showed less total BrdU uptake than BPs cultured in DMEM with matching serum concentration. In addition, while SC division was evident in the abneural region in both media types, substantial cell division in the neural region was only noted in BPs cultured in DMEM. These findings suggest that BME/EBSS lacks a component that promotes or permits SC division in the neural region. Serum inhibited SC division at the highest concentration used in this study-10\%. SC division and HC regeneration were robust in serum-free cultures, similar to what has been described for avian vestibular SC cultures (Warchol and Corwin 1993). This property enables the use of defined media for future studies.

\section{Hair cells are regenerated by mitotic and nonmitotic means}

Many HCs were regenerated in streptomycin-treated BPs after 8 days in culture, but the majority of new HCs were not labeled for BrdU despite its continual presence in culture. Significant numbers of new HCs were regenerated when SC division was blocked, and there was an accompanying decrease in cell density in the BP. These observations suggest many HCs were regenerated by direct transdifferentiation, during which an SC converts phenotypically into an HC without dividing. Further evidence for this phenomenon, which was proposed in the mid-1990s (Baird et al. 1996; Adler and Raphael 1996; Roberson et al. 1996), was recently provided by the observation that SCs upregulate the HC-specific protein Atoh1 shortly after HC damage is initiated in chickens in vivo (Cafaro et al. 2007). We provide additional evidence for "transitional" cells here. However, it remains possible that some new HCs arise from migration of precursor cells from nonsensory regions into the region containing HCs and SCs. Cotanche et al. (1995) showed that hyaline cells, which are located outside the BP along its abneural edge, migrated into the $\mathrm{BP}$ following extreme noise damage, but these cells did not differentiate into new HCs. Hyaline cells, or some other as-of-yet identified population of epithelial cells located outside the BP, may contribute to the formation of some new HCs after drug damage.

It was somewhat surprising that most HCs regenerated after 8 days in culture lacked BrdU labeling. This may occur because HCs formed by mitosis emerge and differentiate later in culture than those formed by direct transdifferentiation. In support of this, we found that BrdU-negative HCs appeared more 
highly differentiated (had developed stereociliary bundles) at 8 days than BrdU-positive HCs. In addition, during in vivo avian auditory $\mathrm{HC}$ regeneration, direct transdifferentiation precedes cell division by $24-48 \mathrm{~h}$ (Roberson et al. 2004; Cafaro et al. 2007). Alternative explanations for these findings are that differentiation of postmitotic cells into HCs is delayed in culture and/ or that incorporated BrdU specifically delays or inhibits HC differentiation. In support of this latter possibility, we found that relatively high concentrations of BrdU inhibit differentiation of myosinVI immunoreactivity in HCs, in a dose-dependent manner. A similar finding has been reported in other tissue types (e.g., Levitt and Dorfman 1972; Tapscott et al. 1989). The mechanism by which BrdU suppresses cellular differentiation is not well understood. BrdU treatment has early effects on gene transcription (e.g., Minagawa et al. 2004), and inhibition of key regulatory genes would have a profound effect on differentiation of some cellular features (e.g., Tapscott et al. 1989).

We have shown that higher BrdU concentrations are more effective reporters of SC division than lower concentrations, yet they inhibit $\mathrm{HC}$ differentiation. These findings demonstrate that $\mathrm{BrdU}$ is a flawed tool for quantifying HC differentiation and cellular proliferation in a single experiment. Therefore, to accurately determine the relative contributions of mitotic regeneration and direct transdifferentiation to $\mathrm{HC}$ regeneration, different measures of cell division or differentiation must be selected.

\section{Contribution of direct transdifferentiation toward morphological and functional recovery}

Basilar papillae in which SC division was blocked formed fewer new HCs than BPs in which SC division occurred freely. This suggests that the rate of direct transdifferentiation could not be increased so as to maintain the rate of $\mathrm{HC}$ regeneration at normal levels and that SC division was also required. A 28\% depletion of epithelial cells accompanied HC regeneration in the absence of SC division, which may be one factor preventing a compensatory increase in direct transdifferentiation. Nonetheless, HCs regenerated by direct transdifferentiation showed advanced signs of maturation, including development of stereociliary bundles and rapid uptake of FM1-43, suggesting that they likely contribute significantly to functional recovery. Although this has not been tested in live chickens, a study by Izumikawa et al. (2005) supports this interpretation. Misexpression of atoh1 in SCs of the damaged organ of Corti in mature guinea pigs appears to trigger the reappearance of HCs inside and outside the organ of Corti, independent of SC division, and to a partial recovery of auditory thresholds compared to control mice.

\section{Summary}

Media type, streptomycin treatment, and serum concentration have significant effects on SC division in the chicken BP in organotypic cultures. Large numbers of new HCs are formed and reach advanced stages of differentiation in streptomycin-treated cultures, demonstrating that this culture method is well suited to study factors regulating avian $\mathrm{HC}$ regeneration. However, an important caveat to consider is that high concentrations of BrdU inhibit differentiation of new HCs. Large numbers of HCs are regenerated in streptomycin-treated cultures despite blockade of SC division, demonstrating that direct transdifferentiation is a significant mechanism of new $\mathrm{HC}$ production in the injured $\mathrm{BP}$.

\section{ACKNOWLEDGMENTS}

We would like to thank Glen MacDonald for assistance with imaging and quantitative analyses, Kevin Whitham and Brandon Warren for assistance with computing, and Dr. Edwin Rubel for sharing laboratory facilities. We are appreciative of Drs. Jane Johnson (Southwestern Texas Medical University) and Guy Richardson (University of Sussex) for sharing antibodies to Atoh1 and HCA, respectively. We are also thankful toward Drs. Elizabeth Oesterle and Allison Coffin (at the UWA) for critical comments on the manuscript. This work was supported by the Otolaryngology/Head and Neck Surgery Department and the Virginia Merrill Bloedel Hearing Research Center at the UWA, NIDCD grants DC03696 (R01) and DC04661 (P30), NICHD grant HD002274 (P30), the Deafness Research Foundation, the National Organization for Hearing Research, and the Hearing Regeneration Initiative.

\section{REFERENCES}

AdLer HJ, RAPHAEL Y (1996) New hair cells arise from supporting cell conversion in the acoustically damaged chick inner ear. Neurosci Lett 205:17-20

Adler HJ, Komeda M, Raphael Y (1997) Further evidence for supporting cell conversion in the damaged avian basilar papilla. Int J Dev Neurosci 15:375-385

Baird RA, Steyger PS, Schuff N (1996) Mitotic and nonmitotic hair cell regeneration in the bullfrog vestibular otolith organs. Ann N Y Acad Sci 781:59-70

Baird RA, Burton MD, Fashena DS, Naeger RA (2000) Hair cell recovery in mitotically blocked cultures of the bullfrog saccule. Proc Natl Acad Sci USA 97:11722-11729

Bartolami S, Goodyear R, Richardson G (1991) Appearance and distribution of the $275 \mathrm{kD}$ hair-cell antigen during development of the avian inner ear. J Comp Neurol 314:777-788

Bermingham-McDonogh O, Rubel EW (2003) Hair cell regeneration: winging our way towards a sound future. Curr Opin Neurobiol 13:119-126

Bhave SA, Stone JS, Rubel EW, Coltrera MD (1995) Cell cycle progression in gentamicin-damaged avian cochleas. J Neurosci 15:4618-4628 
Bhave SA, Oesterle EC, Coltrera MD (1998) Macrophage and microglia-like cells in the avian inner ear. J Comp Neurol 398:241-256

Cafaro J, Lee GS, Stone JS (2007) Atoh1 expression defines activated progenitors and differentiating hair cells during avian hair cell regeneration. Dev Dyn 236:156-170

Cheng AG, Cunningham LL, Rubel EW (2003) Hair cell death in the avian basilar papilla: characterization of the in vitro model and caspase activation. J Assoc Res Otolaryngol 4:91-105

CoRwin JT, Cotanche DA (1988) Regeneration of sensory hair cells after acoustic trauma. Science 240:1772-1774

Cotanche DA, Messana EP, Ofsie MS (1995) Migration of hyaline cells into the chick basilar papilla during severe noise damage. Hear Res 9:148-159

Cunningham LL, Cheng AG, Rubel EW (2002) Caspase activation in hair cells of the mouse utricle exposed to neomycin. J Neurosci 22:8532-8540

Daudet N, Gibson R, Shang J, Bernard A, Lewis J, Stone J (2009) Notch regulation of progenitor cell behavior in quiescent and regenerating auditory epithelium of mature birds. Dev Biol 326:86-100

Duncan LJ, Mangiardi DA, Matsui Ji, Anderson JK, McLaughlinWilliamson K, Cotanche DA (2006) Differential expression of unconventional myosins in apoptotic and regenerating chick hair cells confirms two regeneration mechanisms. J Comp Neurol 499:691-701

Frenz DA, Yoo H, Liu W (1998) Basilar papilla explants: a model to study hair cell regeneration-repair and protection. Acta Otolaryngol 118:651-659

Gale Je, Marcotti W, Kennedy HJ, Kros CJ, Richardson GP (2001) FM1-43 dye behaves as a permeant blocker of the hair-cell mechanotransducer channel. J Neurosci 21:7013-7025

Gale Je, Meyers JR, Periasamy A, Corwin JT (2002) Survival of bundleless hair cells and subsequent bundle replacement in the bullfrog's saccule. J Neurobiol 50:81-92

GÉLÉOC GS, Holt JR (2003) Developmental acquisition of sensory transduction in hair cells of the mouse inner ear. Nat Neurosci 6:1019-1020

Goodyear R, Richardson G (1997) Pattern formation in the basilar papilla: evidence for cell rearrangement. J Neurosci 17:6289-6301

Goodyear R, Killick R, Legan PK, Richardson GP (1996) Distribution of beta-tectorin mRNA in the early posthatch and developing avian inner ear. Hear Res 96:167-178

GratzNER HG (1982) Monoclonal antibody to 5-bromo- and 5iododeoxyuridine: a new reagent for detection of DNA replication. Science 218:474-475

HaShino E, SALVI R (1993) Changing patterns of DNA replication in the noise-damaged chick cochlea. J Cell Science 105:23-31

Hashino E, Tanaka Y, Sokabe M (1991) Hair cell damage and recovery following chronic application of kanamycin in the chick cochlea. Hear Res 52:356-368

IzumikaWa M, Minoda R, KaWamoto K, Abrashikin KA, Swiderski DL, Dolan DF, Brough DE, Raphael Y (2005) Auditory hair cell replacement and hearing improvement by Atoh1 gene therapy in deaf mammals. Nat Med 11:271-276

Janas JD, Cotanche DA, Rubel EW (1995) Avian cochlear hair cell regeneration: stereological analyses of damage and recovery from a single high dose of gentamicin. Hear Res 92:17-29

Levitt D, Dorfman A (1972) The irreversible inhibition of differentiation of limb-bud mesenchyme by bromodeoxyuridine. Proc Natl Acad Sci USA 69:1253-1257

LIPPE WR, WESTBRoOK EW, Ryals BM (1991) Hair cell regeneration in the chicken cochlea following aminoglycoside toxicity. Hear Res 56:203-210

Mangiardi DA, McLaughlin-Williamson K, May Ke, Messana EP, Mountain DC, Cotanche DA (2004) Progression of hair cell ejection and molecular markers of apoptosis in the avian cochlea following gentamicin treatment. J Comp Neurol 475:1-18

Matsui JI, OGilvie JM, Warchol ME (2002) Inhibition of caspases prevents ototoxic and ongoing hair cell death. J Neurosci 22:1218-1227

Meyers JR, MacDonald RB, Duggan A, Lenzi D, Standaert DG, Corwin JT, Corey DP (2003) Lighting up the senses: FM1-43 loading of sensory cells through nonselective ion channels. J Neurosci 23:4054-4065

Minagawa S, Nakabayashi K, Fuji M, Scherer SW, Ayusawa D (2004) Functional and chromosomal clustering of genes responsive to 5-bromodeoxyuridine in human cells. Exp Gerontol 39:10691078

Morest DK, Cotanche DA (2004) Regeneration of the inner ear as a model of neural plasticity. J Neurosci Res 78:455-460

Navaratnam DS, Su HS, Scott SP, Oberholtzer JC (1996) Proliferation in the auditory receptor epithelium mediated by a cyclic AMPdependent signaling pathway. Nat Med 2: 1136-1139

Oesterle EC, Tsue TT, Reh TA, Rubel EW (1993) Hair-cell regeneration in organ cultures of the postnatal chicken inner ear. Hear Res 70:85-108

Pedrali-Noy G, Spadari S, Miller-Faurès A, Miller aO, Kruppa J, KocH G (1980) Synchronization of HeLa cell cultures by inhibition of DNA polymerase alpha with aphidicolin. Nucleic Acids Res 8:377-387

RAPHAEL Y (1992) Evidence for supporting cell mitosis in response to acoustic trauma in the avian inner ear. J Neurocytol 21:663-671

Roberson DW, KreIg CS, Rubel EW (1996) Light microscopic evidence that direct transdifferentiation gives rise to new hair cells in regenerating avian auditory epithelium. Aud Neuroscience 2:195-205

Roberson DW, Alosi JA, Cotanche DA (2004) Direct transdifferentiation gives rise to the earliest new hair cells in regenerating avian auditory epithelium. J Neurosci Res 78:461-471

Ryals BM, Rubel EW (1988) Hair cell regeneration after acoustic trauma in adult Coturnix quail. Science 240:1774-1776

Si F, Brodie H, Gillespie PG, Vazquez Ae, Yamoah EN (2003) Developmental assembly of transduction apparatus in chick basilar papilla. J Neurosci 23:10815-10826

Stone JS, Cotanche DA (1994) Identification of the timing of S phase and the patterns of cell proliferation during hair cell regeneration in the chick cochlea. J Comp Neurol 341:50-67

Stone JS, Cotanche DA (2007) Hair cell regeneration in the avian auditory epithelium. Int J Dev Biol 51:633-647

Stone JS, Rubel EW (2000) Temporal, spatial, and morphologic features of hair cell regeneration in the avian basilar papilla. J Comp Neurol 417:1-16

Stone JS, Leaño SG, BaKer LP, Rubel EW (1996) Hair cell differentiation in chick cochlear epithelium after aminoglycoside toxicity: in vivo and in vitro observations. J Neurosci 16:6157-6174

Stone JS, Choi YS, Woolley SM, Yamashita H, Rubel EW (1999) Progenitor cell cycling during hair cell regeneration in the vestibular and auditory epithelia of the chick. J Neurocytol 28:863-876

Tapscott SJ, Lassar AB, Davis RL, Weintraub H (1989) 5-Bromo-2'deoxyuridine blocks myogenesis by extinguishing expression of MyoD1. Science 245:532-536

TAYLOR RR, Forge A (2005) Hair cell regeneration in sensory epithelia from the inner ear of a urodele amphibian. J Comp Neurol 484:105-120

WARCHOL ME, CORWIN JT (1993) Supporting cells in avian vestibular organs proliferate in serum-free culture. Hear Res 71:28-36

WARCHOL ME, CoRWIN JT (1996) Regenerative proliferation in organ cultures of the avian cochlea: identification of the initial progenitors and determination of the latency of the proliferative response. J Neurosci 16:5466-5477 